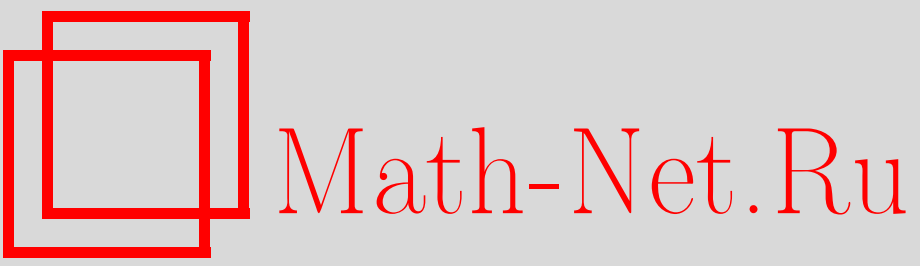

А. Д. Брюно, Автомодельные решения и степенная геометрия, УМH, 2000, том 55, выпуск 1, 3-44

DOI: https://doi.org/10.4213/rm248

Использование Общероссийского математического портала Math-Net.Ru подразумевает, что вы прочитали и согласны с пользовательским соглашением

http://www.mathnet.ru/rus/agreement

Параметры загрузки:

IP : 54.224 .60 .19

26 апреля 2023 г., $15: 31: 43$ 
УДК 517.9

\title{
АВТОМОДЕЛЬНЫЕ РЕШЕНИЯ И СТЕПЕННАЯ ГЕОМЕТРИЯ
}

\author{
А. Д. БРюно
}

\begin{abstract}
Сначала идеи и алгоритмы степенной геометрии применяются для изучения одного дифференциального уравнения в частных производных без параметров. Каждому дифференциальному моному ставится в соответствие точка в $\mathbb{R}^{n}-$ его векторный показатель степени. Дифференциальному уравнению ставится в соответствие его носитель - множество векторных показателей степени его мономов. Показывается, как по носителю уравнения с помощью линейной алгебры вычисляются виды его автомодельных решений. В качестве примеров рассматриваются уравнения процесса горения без источника и с источником. Для квазиоднородного обыкновенного дифоференциального уравнения этот подход позволяет также понижать порядок и упрощать некоторые граничные задачи. Затем формулируются обобщения для системы уравнений. Кроме того, дается классификация уровней сложности задач степенной геометрии. Эта классификация содержит 4 уровня и основана на сложности геометрических объектов, соответствующих той или иной задаче в пространстве показателей степеней. Приводится сравнительный обзор этих объектов и основанных на них способов анализа решений систем алгебраических уравнений, систем обькновенных дифференциальных уравнений и систем уравнений в частных производных. Указываются некоторые работы, в которых эффективно применялись методы степенной геометрии.

Библиограффия: 110 названий.
\end{abstract}

\section{СОДЕРЖАНИЕ}

Введение

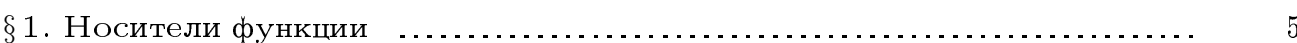

$\S 2$. Носители дифференциального полинома ........................ 6

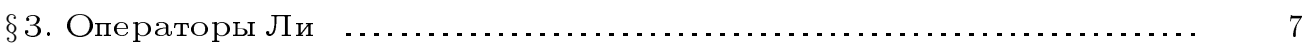

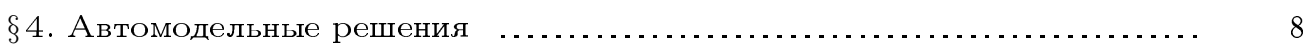

$\S 5$. Степенное преобразование ................................... 14

$\S 6$. Логарифмическое преобразование ............................. 19

$\S 7$. Обыкновенное дифференциальное уравнение ................... 21

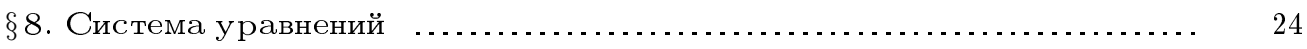

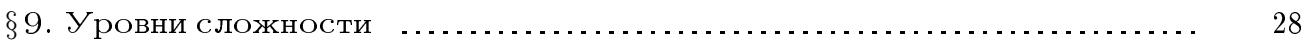

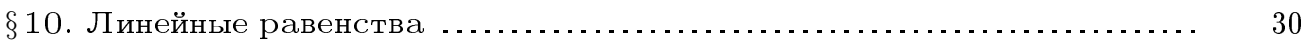

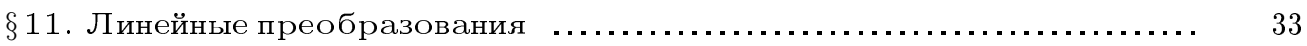

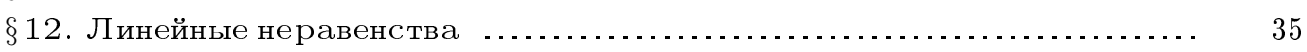

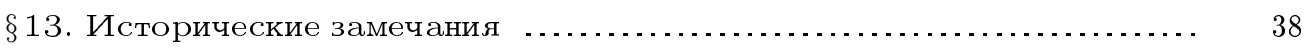

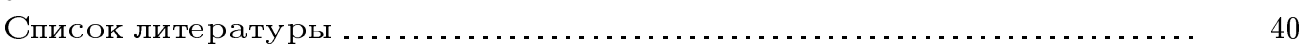

(C) А. Д. Брюно 2000 


\section{Введение}

Степенная геометрия - это новое исчисление, развивающее дифференциальное исчисление и нацеленное на нелинейные задачи. Основная ее концепция заключается в изучении нелинейных задач не в исходных координатах, а в логарифмах этих координат. Тогда многим свойствам и соотношениям, нелинейным в исходных координатах, можно поставить в соответствие определенные линейные соотношения. Алгоритмы степенной геометрии основаны на этих линейных соотношениях; они позволяют упрощать уравнения, разрешать их особенности, выделять их первые приближения и находить либо их решения, либо асимптотики их решений. После одного шага таких упрощающих преобразований можно легко получить степенные решения или степенные асимптотики решений. После кратного применения этих алгоритмов можно легко получить решения или их асимптотики, содержашие кратные логарифмы и экспоненты. Этот подход позволяет также вычислять асимптотические и локальные разложения решений. Алгоритмы степенной геометрии применимы к уравнениям разных типов: алгебраическим, обыкновенньм дифференциальным и в частных производных, а также - к системам таких уравнений. Элементарным введением в степенную геометрию для алгебраических уравнений и для обыкновенных дифференциальных уравнений служат соответственно главы I и II книги [18]. Более продвинутое изложение для всех типов уравнений см. в книге [30].

Здесь идеи и алгоритмы степенной геометрии сначала применяются для одного дифференциального уравнения в частных производных без параметров. А именно, каждому дифференциальному моному ставится в соответствие точка в $\mathbb{R}^{n}$ - его векторньй показатель степени. Дифференциальному полиному, который является конечной суммой дифференциальных мономов, ставится в соответствие его носитель множество векторных показателей степени его мономов. Аффинная оболочка носителя называется сверхносителем, а ее размерность - размерностью дифференциального полинома. Каждому дифференциальному полиному соответствует дифференциальное уравнение, полученное приравниванием этого полинома нулю. Такое уравнение имеет носитель, сверхноситель и размерность, определяемые по полиному. Если размерность меншше $n$, то дифференциальный полином является квазиоднородным и соответствуюшее уравнение имеет квазиоднородные (автомодельные) решения. Такое решение определяется функцией от меньшего числа независимых переменных. Здесь показано, как по носителю уравнения с помощью линейной алгебры вычисляются виды его автомодельных решений и как упрошается само уравнение с помошью степенного преобразования (линейного в логарифмах координат). В качестве примеров рассмотрены уравнения процесса горения без источника и с источником.

Для квазиоднородного обыкновенного дифференциального уравнения этот подход позволяет находить его степенные решения, понижать порядок и упрощать некоторые граничные задачи. Сформулированы обобщения для системы дифференциальных уравнений.

Кроме того, дана классификация уровней сложности задач степенной геометрии. Эта классификация содержит 4 уровня и основана на сложности геометрических объ-

Работа выполнена при финансовой поддержке Российского фонда фундаментальных исследований (грант № 99-01-01063). 
ектов, соответствующих той или иной задаче в пространстве показателей степеней. Дан сравнительньй обзор этих объектов и основанных на них способов анализа решений системы алгебраических уравнений, системы обыкновенных дифференциальных уравнений и системы уравнений в частных производных. Указаны некоторые работы, в которых эффективно применялись методы степенной геометрии.

Для экономии места векторы записьваются как матрипы-строки, но в матричных операциях они предполагаются матрицами-столбцами. Если $A$ - матрица, то $A^{*}-$ транспонированная матрица $A$. Для векторов транспонирование не оговаривается.

\section{$\S 1$. Носители функции}

Пусть $X=\left(x_{1}, \ldots, x_{n}\right) \in \mathbb{K}^{n}$, где $\mathbb{K}$ есть $\mathbb{C}$ или $\mathbb{R}$, а $Q=\left(q_{1}, \ldots, q_{n}\right) \in \mathbb{R}^{n} ;$ через $X^{Q}$ будем обозначать моном $x_{1}^{q_{1}} \cdots x_{n}^{q_{n}}$. Сумме

$$
f(X)=\sum f_{Q} X^{Q} \text { по } Q \in \mathbf{S},
$$

в которой коэффициенты $f_{Q} \in \mathbb{K}$ и все подобные члены приведены, поставим в соответствие множество

$$
\mathbf{S}(f)=\left\{Q: f_{Q} \neq 0\right\}
$$

в пространстве $\mathbb{R}^{n}$. Это множество $\mathbf{S}(f)$ называется носителем суммы (1.1) [18]. Пусть $\mathbf{S S}(f)$ - наименьшее линейное многообразие в $\mathbb{R}^{n}$, содержашее множество $\mathbf{S}(f)$; назовем его сверхносителем суммы (1.1). Число $d(f) \stackrel{\text { def }}{=} \operatorname{dim} \mathbf{S S}(f)$ назовем размерностью суммы (1.1).

Если функция $f(X)$ не является суммой вида (1.1), то она может не иметь точечного носителя (1.2), но может иметь сверхноситель $\mathbf{S S}(f)$. Пусть $d<n$, векторы $B_{1}, \ldots, B_{d} \in \mathbb{R}^{n}$ линейно независимы и вектор $R \in \mathbb{R}^{n}$. Пусть $g\left(y_{1}, \ldots, y_{d}\right)$ - произвольная гладкая функция своих аргументов, причем $\partial g / \partial y_{j} \not \equiv 0, j=1, \ldots, d$. Тогда линейное многообразие в $\mathbb{R}^{n}$

$$
\left\{Q: Q=R+\sum_{j=1}^{d} \mu_{j} B_{j}, \mu_{j} \in \mathbb{R}^{n}\right\}
$$

назовем сверхносителем функиии

$$
f(X)=X^{R} g\left(X^{B_{1}}, \ldots, X^{B_{d}}\right)
$$

и обозначим $\mathbf{S S}(f)$. Для суммы (1.1) оба определения сверхносителя $\mathbf{S S}(f)$ совпадают. Число $d(f) \stackrel{\text { def }}{=} \operatorname{dim} \mathbf{S S}(f)$ назовем размерностью функиии (1.4).

ПримеР 1. Пусть $n=2$ и $f=x_{1}+x_{2}$. Тогда носитель $\mathbf{S}(f)$ состоит из двух точек $E_{1}=(1,0)$ и $E_{2}=(0,1)$, а сверхноситель по первому определению это прямая

$$
q_{1}+q_{2}=1
$$


проходящая через точки $E_{1}$ и $E_{2}$, поэтому $d(f)=1$. Ее нормальньй вектор $N=(1,1)$. Если записать $f$ в виде (1.4) как $f=x_{1}\left(1+x_{1}^{-1} x_{2}\right)$, то $d=1, B_{1}=N$ и по второму определению (1.3) сверхноситель $\mathbf{S S}(f)$ это прямая

$$
\mathbf{S S}(f)=\{Q: Q=(1,0)+\mu(-1,1), \mu \in \mathbb{R}\}
$$

с направляюшим вектором $B_{1}=(-1,1)$. При $\mu=0$ и $\mu=1$ она проходит через точки $E_{1}$ и $E_{2}$ соответственно, т.е. совпадает с прямой (1.5). Записи (1.5) и (1.6) это двойственнье описания [51] одной и той же прямой.

Рассмотрим теперь пространство $\mathbb{R}_{*}^{n}$, двойственное к пространству $\mathbb{R}^{n}$. Так что для $P=\left(p_{1}, \ldots, p_{n}\right) \in \mathbb{R}_{*}^{n}$ и $Q=\left(q_{1}, \ldots, q_{n}\right) \in \mathbb{R}^{n}$ определено скалярное произведение $\langle P, Q\rangle=p_{1} q_{1}+\cdots+p_{n} q_{n}$.

Пусть $\mathbf{K}$ - некоторое линейное многообразие в $\mathbb{R}^{n}$ и $\mathbf{N}=\mathbf{N}(\mathbf{K})$ - максимальное линейное подпространство в $\mathbb{R}_{*}^{n}$, нормальное к многообразию $\mathbf{K}$. Для функции $f$ вида (1.4) вместе со сверхносителем $\mathbf{S S}(f)$ будем рассматривать его нормальное подпространство $\mathbf{N}(f) \stackrel{\text { def }}{=} \mathbf{N}(\mathbf{S S}(f)) \subset \mathbb{R}_{*}^{n}$. Пусть векторы $N_{1}, \ldots, N_{n-d} \in \mathbb{R}_{*}^{n}$ линейно независимы и нормальны к векторам $B_{1}, \ldots, B_{d}$, тогда они образуют базис нормального подпространства $\mathbf{N}(f)$ функции (1.4).

\section{§ 2. Носители дифференциального полинома}

Будем штрихом выделять векторы длины $n-1$. Так, $X^{\prime}=\left(x_{1}, \ldots, x_{n-1}\right)$. Дифференциальныц мономом $а(X)$ в [26], [28], [30; гл. VI] называется произведение степеней координат $X$ и производных вида

$$
\partial^{\left\|L^{\prime}\right\|} x_{n} / \partial x_{1}^{l_{1}} \cdots \partial x_{n-1}^{l_{n-1}},
$$

где $L^{\prime}=\left(l_{1}, \ldots, l_{n-1}\right) \in \mathbb{Z}_{+}^{n-1}$, т.е. все $l_{i} \geqslant 0$ и целые, а $\left\|L^{\prime}\right\|=l_{1}+\cdots+l_{n-1}$. Каждому дифференциальному моному $a(X)$ поставим в соответствие точку $Q(a) \in \mathbb{R}^{n}$ (его векторный показатель степени) по следуюшему правилу: моному const $X^{Q}$ соответствует точка $Q ;$ производной $(2.1)$ соответствует точка

$$
Q=\left(-L^{\prime}, 1\right), \quad \text { т.е. } Q^{\prime}=-L^{\prime}, \quad q_{n}=1 ;
$$

а произведению двух дифференциальных мономов $a(X)$ и $b(X)$ ставится в соответствие точка $Q(a b)=Q(a)+Q(b)$. Конечная сумма дифференциальных мономов

$$
f(X)=\sum_{i=1}^{s} a_{i}(X)
$$

назьвается дифференциальны.м полиномом [26], [28], [30; гл. VI]. Множество точек $\mathbf{S}(f) \stackrel{\text { def }}{=}\left\{Q\left(a_{i}\right), i=1, \ldots, s\right\}$ назьвается носителем дифференциального полинома (2.3). Минимальное линейное многообразие $\mathbf{S S}(f)$, содержащее носитель $\mathbf{S}(f)$, назовем сверхносителем полинома (2.3). Аналогично определяется размерность $d(f) \stackrel{\text { def }}{=} \operatorname{dim} \mathbf{S S}(f)$ и нормальное подпространство $\mathbf{N}(f) \stackrel{\text { def }}{=} \mathbf{N}(\mathbf{S S}(f)) \subset \mathbb{R}_{*}^{n}$. 
ПримеР 2. Рассмотрим одномерное уравнение процесса горения без источников

$$
u_{t}=\left(u^{\sigma} u_{x}\right)_{x}, \quad \sigma=\text { const }>0
$$

для температуры $u$, времени $t$ и единственной пространственной координаты $x$ [72], [90]. Здесь $n=3$,

$$
x_{1}=t, \quad x_{2}=x, \quad x_{3}=u \text {. }
$$

Носитель $\mathbf{S}$ состоит из двух точек $Q_{1}=(-1,0,1)$ и $Q_{2}=(0,-2,1+\sigma)$. Сверхноситель $\mathbf{S S}$ здесь - прямая, проходящая через точку $Q_{1}$ с направляюшим вектором $B=Q_{2}-Q_{1}=(1,-2, \sigma)$, т.е. прямая $\left\{Q: Q_{1}+\mu B, \mu \in \mathbb{R}\right\}$. Следовательно, $d=1$. Нормальное подпространство $\mathbf{N}$ двумерно с базисом $N_{1}=(2,1,0)$ и $N_{2}=(0, \sigma, 2)$.

\section{§ 3. Операторы Ли}

Дифференциальное уравнение $f(X)=0$, соответствуюшее полиному $(2.3)$, допускает оператор Ли

$$
\sum_{i=1}^{n} \lambda_{i} x_{i} \frac{\partial}{\partial x_{i}}
$$

если это уравнение инвариантно относительно замены координат

$$
\widetilde{x}_{i}=x_{i} \mu^{\lambda_{i}}, \quad \mu \in \mathbb{R}, \quad \mu>0, \quad i=1, \ldots, n
$$

т.е. является квазиоднородным (см. [60], [84]).

Теорема 1. Дифференциальное уравнение $f(X)=0$, соответствующее полиному (2.3), допускает оператор Ли (3.1), если вектор $\Lambda=\left(\lambda_{1}, \ldots, \lambda_{n}\right) \in \mathbf{N}(f)$.

ДокАЗАТЕЛЬСТво. При подстановке (3.2) моном $X^{Q}=\mu^{-\langle\Lambda, Q\rangle} \widetilde{X}^{Q}$, производная (2.1)

$$
\partial^{\left\|L^{\prime}\right\|} x_{n} / \partial x_{1}^{l_{1}} \cdots \partial x_{n-1}^{l_{n-1}}=\mu^{-\langle\Lambda, Q\rangle} \partial^{\left\|L^{\prime}\right\|} \widetilde{x}_{n} / \partial \widetilde{x}_{1}^{l_{1}} \cdots \partial \widetilde{x}_{n-1}^{l_{n-1}}
$$

с вектором $Q$, указанньм в (2.2), а произведение мономов и производных приобретает в качестве множителя такую степень $\mu$, которая равна сумме степеней $\mu$, приобретенных каждым из сомножителей. Поэтому при подстановке (3.2) дифференциальньй моном $a(X)$ переходит в моном $a(\widetilde{X}) \mu^{-\langle\Lambda, Q\rangle}$, где $Q=Q(a)$, а дифференциальньй полином (2.3) переходит в

$$
\sum_{i=1}^{s} a_{i}(\widetilde{X}) \mu^{-\left\langle\Lambda, Q_{i}\right\rangle}
$$

где $Q_{i}=Q\left(a_{i}\right)$. Если вектор $\Lambda \in \mathbf{N}(f)$, то все скалярные произведения $-\left\langle\Lambda, Q_{i}\right\rangle$ в (3.3) равны между собой для $i=1, \ldots, s$. Поэтому $f(X)=f(\widetilde{X}) \mu^{-\left\langle\Lambda, Q_{1}\right\rangle}$ и уравнение $f(X)=0$ переходит в себя при подстановке (3.2), т.е. допускает оператор (3.1). Доказательство окончено. 
Если $d \stackrel{\text { def }}{=} d(f)<n$, то $\operatorname{dim} \mathbf{N}=n-d>0$, и уравнение $f(X)=0$ допускает $n-d$ линейно независимых операторов (3.1), которые легко вычисляются по $\mathbf{S}(f)$.

Выражение $x_{n}=\varphi\left(X^{\prime}\right)$ называется решением уравнения $f(X)=0$, если $f\left(X^{\prime}, \varphi\left(X^{\prime}\right)\right) \equiv 0$. Решение называется автомодельным, если оно инвариантно относительно замен координат, образуюших группу Ли [59], [84]. В частности, решение является автомодельным, если оно допускает оператор вида (3.1), т.е. инвариантно относительно замен (3.2). Можно искать такие автомодельные решения $x_{n}=\varphi\left(X^{\prime}\right)$ уравнения $f(X)=0$, которые допускают некоторые из операторов (3.1), допускаемых уравнением. Если решение $x_{n}=\varphi\left(X^{\prime}\right)$ допускает оператор (3.1), то соответствуюший сверхноситель $\mathbf{S S}\left(x_{n}-\varphi\left(X^{\prime}\right)\right)$ нормален к вектору $\Lambda$. Для разности $x_{n}-\varphi\left(X^{\prime}\right)$ сверхноситель обязательно проходит через точку $Q=E_{n}=(0, \ldots, 0,1)$, соответствуюшую члену $x_{n}$, а сверхноситель $\mathbf{S S}\left(\varphi\left(X^{\prime}\right)\right)$ расположен в гиперплоскости $q_{n}=0$. Поэтому сверхноситель $\mathbf{S S}\left(x_{n}-\varphi\left(X^{\prime}\right)\right)$ расположен в гиперплоскости

$$
\left\{Q:\langle\Lambda, Q\rangle=\lambda_{n}\right\}
$$

Она пересекает гиперплоскость $q_{n}=0$ по линейному многообразию

$$
\left\{Q^{\prime}:\left\langle\Lambda^{\prime}, Q^{\prime}\right\rangle=\lambda_{n}\right\}
$$

в котором расположен сверхноситель $\mathbf{S S}\left(\varphi\left(X^{\prime}\right)\right)$. Если решение $x_{n}=\varphi\left(X^{\prime}\right)$ допускает несколько операторов вида $(3.1)$ с векторами $\Lambda_{i}=\left(\lambda_{1 i}, \ldots, \lambda_{n i}\right), i=1, \ldots, m$, то сверхноситель $\mathbf{S S}\left(\varphi\left(X^{\prime}\right)\right)$ лежит в линейном многообразии

$$
\left\{Q^{\prime}:\left\langle\Lambda_{i}^{\prime}, Q^{\prime}\right\rangle=\lambda_{n i}, i=1, \ldots, m\right\}
$$

ПримеР 3 (продолжение примера 2). Уравнение (2.4) допускает операторы (3.1) с $\Lambda=N_{1}$ и $\Lambda=N_{2}$.

\section{§4. Автомодельные решения}

Для суммы (2.3) ее собственной подсуммой $\check{f}$ назовем сумму некоторого числа $s_{1}$ ее слагаемых $a_{i}(X)$, где $0<s_{1} \leqslant s$.

Tеорема 2. Пусть $x_{n}=\varphi\left(X^{\prime}\right)-$ такое решение уравнения $f(X)=0$, соответствующего полиному (2.3), что никакая собственная подсумма $\check{f}$ сумми (2.3) $c \operatorname{dim}(\check{f})<\operatorname{dim}(f)$ не обращается в нуль тождественно на этом решении. Тогда

$$
\mathbf{N}\left(x_{n}-\varphi\left(X^{\prime}\right)\right) \subset \mathbf{N}(f) .
$$


ДокАЗАТЕЛЬСтвО. Пусть вектор $\Lambda \in \mathbf{N}\left(x_{n}-\varphi\left(X^{\prime}\right)\right)$, тогда согласно (3.5) $\lambda_{n}=$ $\left\langle\Lambda^{\prime}, Q^{\prime}\right\rangle$, где $Q^{\prime}$ пробегает сверхноситель функции $\varphi\left(X^{\prime}\right)$. При подстановке (3.2) дифоференциальный полином (2.3) приобретает вид (3.3). Поскольку $x_{n}=\varphi\left(X^{\prime}\right)-$ peшение уравнения $f(X)=0$, инвариантное при подстановке $(3.2)$, т.е. $\widetilde{x}_{n}=\varphi\left(\widetilde{X}^{\prime}\right)$ является тем же решением, то на нем

$$
\sum_{i=1}^{s} a_{i}(\tilde{X}) \mu^{-\left\langle\Lambda, Q_{i}\right\rangle}=0 .
$$

Пусть различные значения скалярных произведений $\left\langle\Lambda, Q_{i}\right\rangle$ суть $c_{1}<c_{2}<\cdots<c_{t}$. Обозначим через $I_{r}$ набор всех таких индексов $i$, что $\left\langle\Lambda, Q_{i}\right\rangle=c_{r}, r=1, \ldots, t$. Определим дифференциальные полиномы

$$
f_{r}(X) \stackrel{\text { def }}{=} \sum a_{i}(X) \quad \text { по } i \in I_{r}, \quad r=1, \ldots, t .
$$

Тогда равенство (4.2) принимает вид

$$
\sum_{r=1}^{t} \mu^{-c_{r}} f_{r}(\widetilde{X})=0 .
$$

Поскольку это равенство справедливо для всех $\mu>0$, то на нашем решении выполняются равенства

$$
f_{r}(\widetilde{X})=0, \quad r=1, \ldots, t,
$$

и оно является решением этой системы уравнений. По построению полиномов $f_{r}(X)$ вектор $\Lambda \in \mathbf{N}\left(f_{r}\right)$ для всех $r=1, \ldots, t$. Следовательно,

$$
\Lambda \in \mathbf{N}\left(f_{1}\right) \cap \cdots \cap \mathbf{N}\left(f_{t}\right) .
$$

Если $d\left(f_{r}\right)=d(f)$ для какого-то $r$, то $\mathbf{N}\left(f_{r}\right)=\mathbf{N}(f)$ и свойство (4.1) следует из (4.3). По условию теоремы $d\left(f_{r}\right)=d(f)$ для всех $r=1, \ldots, t$. Поэтому свойство (4.1) выполнено. Если же $d\left(f_{r}\right)<d(f)$ для всех $r$ и пересечение (4.3) не лежит в $\mathbf{N}(f)$, то включение (4.1) может нарушаться (см. ниже пример 6). Доказательство окончено.

ПримеР 4 (продолжение примеров 2 и 3 ). Рассмотрим уравнение (2.4) в координатах (2.5). Если через точку $E_{3}=(0,0,1)$ провести прямую, параллельную $\mathbf{S S}(f)$, т.е. с направляюшим вектором $B=(1,-2, \sigma)$, то эта прямая пересекает плоскость $q_{3}=0$ в точке $Q_{4}=(-1 / \sigma, 2 / \sigma, 0)$. Свойство (4.1) означает, что для решения $x_{3}=\varphi\left(x_{1}, x_{2}\right)$ уравнения (2.4) сверхноситель функции $\varphi$ на плоскости $q_{3}=0$ либо состоит из точки $Q_{4}$, либо является прямой, проходящей через эту точку, либо совпадает со всей плоскостью. Поэтому решения, относящиеся к первым двум случаям, можно искать в виде

$$
x_{3}=c w_{1}^{-1 / \sigma} w_{2}^{2 / \sigma},
$$

где $w_{i}=c_{i}+\delta_{i} x_{i}, c_{i}=$ const, $\delta_{i}= \pm 1, i=1,2$. В первом случае $c=$ const и для $c$ получаем "алгебраическое" уравнение $-\delta_{1}=2(2+\sigma) c^{\sigma} / \sigma$. Во втором случае $c-$ 
функция от произвольного монома $w_{1}^{b} w_{2}$. На плоскости $q_{3}=0$ прямая, проходяшая через точку $Q_{4}$ с направляющим вектором $(b, 1,0)$, имеет вид $\left(q_{1}, q_{2}\right)=(-1 / \sigma, 2 / \sigma)+$ $\mu(b, 1), \mu \in \mathbb{R}$. Она пересекает ось $q_{1}$ при $\mu=-2 / \sigma$ в точке с $q_{1}=-(1+2 b) / \sigma$. Поэтому эту прямую можно также записать в виде

$$
\left(q_{1}, q_{2}\right)=(-(1+2 b) / \sigma, 0)+\mu(b, 1),
$$

а автомодельное решение (4.4), у которого сверхноситель правой части расположен на этой прямой, можно записать в виде

$$
x_{3}=w_{1}^{-(1+2 b) / \sigma} v(\xi), \quad \xi=w_{1}^{b} w_{2} .
$$

Подставляя это выражение в уравнение $(2.4)$ и сокращая на $w_{1}^{-1-(1+2 b) / \sigma}$, для функции $v(\xi)$ получаем обыкновенное дифференциальное уравнение

$$
\delta_{1}\left[b v^{\prime} \xi-(1+2 b) v / \sigma\right]=v^{\sigma} v^{\prime \prime}+\sigma v^{\sigma-1} v^{\prime 2}
$$

где штрих означает производную по $\xi$. В обоих случаях решение (4.4) является автомодельным, но имеет разную размерность $d\left(x_{3}-\varphi\right)$ : единицу в первом случае и двойку во втором случае. При $\delta_{1}=-1$ и $c_{1}>0$ решение (4.4) в первом случае является решением с обострением, т.е. уходит в бесконечность за конечноевремя [90]. Эти решения изучались в [90; гл. III] в $\S 1$ и $§ 2$ для первого и второго случаев соответственно.

ПримеР 5. Рассмотрим уравнение процесса горения с источником [72], [90]

$$
u_{t}=\left(u^{\sigma} u_{x}\right)_{x}+u^{\beta}, \quad \sigma=\text { const }>0, \quad \beta=\text { const }>0 .
$$

Здесь $n=3$. В обозначениях (2.5) носитель $\mathbf{S}$ для этого уравнения состоит из трех точек $Q_{1}=(-1,0,1), Q_{2}=(0,-2, \sigma+1), Q_{3}=(0,0, \beta)$. Составим разности

$$
\begin{aligned}
& Q_{2}-Q_{1}=(1,-2, \sigma) \stackrel{\text { def }}{=} B_{1}, \\
& Q_{3}-Q_{1}=(1,0, \beta-1) \stackrel{\text { def }}{=} B_{2}
\end{aligned}
$$

и вычислим их векторное произведение $N \stackrel{\text { def }}{=}\left[B_{1}, B_{2}\right]=(2-2 \beta, 1+\sigma-\beta, 2)$. Поскольку $N \neq 0$ при любых значениях показателей $\sigma$ и $\beta$, то векторы $B_{1}$ и $B_{2}$ линейно независимы и размерность уравнения $d=2$. Его сверхноситель в $\mathbb{R}^{3}$ является плоскостью с нормальным вектором $N$. Свойство (4.1) означает, что сверхноситель решения $x_{3}=\varphi\left(w_{1}, w_{2}\right)$ либо совпадает с $\mathbb{R}^{3}$, либо является плоскостью с нормальным вектором $N$. Поскольку плоскость $\mathbf{S S}\left(x_{3}-\varphi\right)$ проходит через точку $E_{3}=(0,0,1)$, то ее уравнение $(3.4)$ есть $2(1-\beta) q_{1}+(1+\sigma-\beta) q_{2}+2 q_{3}=2$, и она пересекает плоскость $q_{3}=0$ по прямой $(3.5)$, т.е.

$$
2(1-\beta) q_{1}+(1+\sigma-\beta) q_{2}=2
$$

Рассмотрим сначала случай

$$
\beta \neq 1
$$


В этом случае прямая (4.6) пересекает ось $q_{1}$ в точке $q_{1}=1 /(1-\beta)$. Следовательно, уравнение (4.5) имеет автомодельное решение вида

$$
u=w_{1}^{1 /(1-\beta)} \psi\left(w_{1}^{\kappa} w_{2}\right), \quad \kappa=-\frac{1+\sigma-\beta}{2(1-\beta)},
$$

где вектор $(\kappa, 1,0)$ является направляюшим для прямой $(4.6), w_{1}=c_{1}+\delta_{1} t$, $w_{2}=c_{2}+\delta_{2} x, c_{1}, c_{2}=$ const, $\delta_{i}= \pm 1$. Функция $\psi(\xi)$ удовлетворяет обыкновенному дифференшиальному уравнению

$$
\delta_{1}\left(\psi /(1-\beta)+\kappa \psi^{\prime} \xi\right)=\psi^{\prime \prime} \psi^{\sigma}+\sigma \psi^{\prime 2} \psi^{\sigma-1}+\psi^{\beta},
$$

где $\xi=w_{1}^{\kappa} w_{2}$ и штрих означает дифференцирование по $\xi$. В [72], [90] асимптотики и локальные свойства ограниченных решений уравнения (4.9) либо пространно изучаются, либо даются без объяснений для различных значений показателей $\sigma>0$ и $\beta>1$, но их можно просто изучить методами гл. VI [30] (см. конец этого примера). Если при $w_{1} \rightarrow 0$ функция $\psi(\xi)$ не стремится к нулю, то при $\beta>1$ решение (4.8) стремится к бесконечности, т.е. при $c_{1}>0$ и $\delta_{1}=-1$ оно является решением с обострением [72], [90]. При $\beta<1$ и ограниченной функции $\psi(\xi)$ решение (4.8) не стремится к бесконечности при конечном $t$.

Рассмотрим теперь случай

$$
\beta=1 .
$$

В этом случае уравнение прямой (4.6) есть $q_{2}=2 / \sigma$. Поэтому уравнение (4.5) в случае (4.10) имеет автомодельное решение

$$
x_{3}=v(t) w_{2}^{2 / \sigma} .
$$

Уравнение (4.5) для функции $v$ дает обыкновенное дифференциальное уравнение

$$
\dot{v}=\omega v^{\sigma+1}+v, \quad \omega=2(2+\sigma) / \sigma^{2}=\text { const },
$$

где точка означает дифференцирование по $t$. Его решения суть

$$
\ln \frac{v}{\left(\omega v^{\sigma}+1\right)^{1 / \sigma}}=t+c,
$$

где $c$ - произвольная постоянная. При $v \rightarrow \infty$ левая часть последнего равенства имеет асимптотику

$$
-\frac{1}{\sigma}\left(\ln \omega+\frac{1}{\omega v^{\sigma}}\right) .
$$

Поэтому при $v \rightarrow \infty$ имеем $v^{-\sigma} \approx-\sigma \omega t+c_{1}, c_{1}=$ const, т.e. $v \approx\left(c_{1}-\sigma \omega t\right)^{-1 / \sigma}$. Это означает, что в случае (4.10) решение (4.11) уравнения (4.5) является решением с обострением.

Этот результат можно получить другим способом: методами гл. VI [30]. В этом случае $n=2$. На плоскости $q_{1}, q_{2}$ носитель уравнения (4.12) состоит из трех точек 
$(-1,1),(0, \sigma+1),(0,1)$. Их выпуклая оболочка является треугольником с вершинами в этих точках. Граница треугольника состоит из трех вершин и трех ребер. Ребро треугольника, соединяюшее первые две вершины, имеет нормальным конусом луч, натянутьй на вектор $(-\sigma, 1)$. Этому ребру соответствует укороченное уравнение $\dot{v}=\omega v^{\sigma+1}$. Оно имеет степенное решение $v=\alpha w_{1}^{-1 / \sigma}$, где $-\delta_{1} / \sigma=\omega \alpha$, т.е. $\alpha=-\delta_{1} /(\sigma \omega)$. Это решение укороченного уравнения является асимптотикой решения полного уравнения при $w_{1} \rightarrow 0$ и $v \rightarrow \infty$, ибо в базисном векторе $(-\sigma, 1)$ нормального конуса первая координата отрищательна, а вторая положительна.

Третий способ - применить методы гл. III [30]. В этом случае $n=1$. Запишем уравнение $(4.12)$ в виде $(\ln v)=\omega v^{\sigma}+1$. Тогда носитель этой системы состоит из двух точек $q_{1}=\sigma$ и $q_{1}=0$. Их выпуклая оболочка это отрезок $[0, \sigma]$, ибо $\sigma>0$. Решениям с $v \rightarrow \infty$ соответствует правая вершина этого отрезка, т.е. точка $q_{1}=\sigma$. Ей соответствует укороченная система $(\ln v)=\omega v^{\sigma}$. Далее как во втором способе.

Четвертый способ получения решения с обострением предложил С. А. Посашков. При $\beta=1$ замена $\widetilde{u}=u e^{-t}, \tilde{t}=\left(e^{\sigma t}-1\right) / \sigma$ переводит уравнение $(4.5)$ в уравнение $(2.4)$ (см. [90; гл. II, $\S 7$, п. 1]). При этом решениям с обострением (4.4) уравнения (2.4) соответствуют решения с обострением уравнения (4.5). В частности, можно взять решение (4.4), относящееся к первому (алгебраическому) случаю.

Теоремы 1 и 2 дают такой алгоритм для нахождения всех автомодельных решений, которьй существенно проще, чем традищионный способ вычисления операторов Ли, допускаемых данным уравнением [59], [84]. Проверка включения (4.1) для результатов, приведенных в [60], выявила там ряд опечаток и неточностей. Некоторые из них обсуждаются ниже в примере 6. Судя по [60], среди всех симметрий дифференциальных уравнений примерно $70 \%$ составляют симметрии, соответствующие операторам вида (3.1), т.е. степенные и логарифмические.

Пример 6. Проверка включения (4.1) для результатов, приведенных в [60], выявила там следуюшие три случая его нарушения.

1. Страница 120, строка 12 [60] (см. также [83]). Уравнение $u_{t}=\left(k(u) u_{x}\right)_{x}$ при $k=u^{-4 / 3}$, решение $u=x^{-3} f(t)$. Здесь $n=3, x_{1}=t, x_{2}=x, x_{3}=u$. Носитель уравнения состоит из двух точек $Q_{1}=(-1,0,1)$ и $Q_{2}=(0,-2,-1 / 3)$, его размерность $d=1$. Направляющий вектор сверхносителя уравнения есть $B \stackrel{\text { def }}{=} Q_{2}-$ $Q_{1}=(1,-2,-4 / 3)$. Если $f(t)$ - произвольная функция, то сверхноситель решения параллелен вектору $E_{1}=(1,0,0)$; кроме того, он проходит через точки $E_{3}=(0,0,1)$ и $Q_{3}=(0,-3,0)$, т.е. параллелен вектору $E_{3}-Q_{3}=(0,3,1) \stackrel{\text { def }}{=} B_{2}$. Векторное произведение $\left[E_{1}, B_{2}\right]=(0,-1,3) \stackrel{\text { def }}{=} N$ является нормальным вектором к сверхносителю решения. Скалярное произведение $\langle N, B\rangle=-2 \neq 0$, т.е. свойство (4.1) не выполнено. Найдем уравнение для функции $f(t)$. Подставляя указанное решение в исходное уравнение, получаем для $f(t)$ уравнение $f^{\prime}=0$. Следовательно, $f(t)=$ const. В этом случае носитель решения состоит из двух точек $E_{3}$ и $Q_{3}$, а направляющий вектор сверхносителя решения есть $B_{2}$. Он не коллинеарен вектору $B$, и свойство (4.1) теоремы 2 не выполнено. Но на этом решении условие теоремы 2 также не выполнено, ибо $u_{t} \equiv 0$ и $\left(u^{-4 / 3} u_{x}\right)_{x} \equiv 0$, т.е. обрашаются в нуль обе части исходного уравнения. Каждая из них имеет нулевую размерность, и для каждой из них свойство (4.1) вьполнено. Это показьвает, что в теореме 2 условие на решение сушественно. 
2. Странища 196, п. 11.10 [60]. Для уравнения

$$
u_{t}+u u_{x}+\beta u_{t t t}=0
$$

указано автомодельное решение

$$
u=t^{2} U\left(t^{-3} x\right) .
$$

Здесь $n=3, x_{1}=t, x_{2}=x, x_{3}=u$. Носитель уравнения (4.13) состоит из трех точек $Q_{1}=(-1,0,1), Q_{2}=(0,-1,2), Q_{3}=(-3,0,1)$. Их разности суть

$$
\begin{aligned}
& B_{1} \stackrel{\text { def }}{=} Q_{2}-Q_{1}=(1,-1,1), \\
& B_{2} \stackrel{\text { def }}{=} Q_{3}-Q_{1}=(-2,0,0) .
\end{aligned}
$$

Векторное произведение $\left[B_{1}, B_{2}\right]=-(0,2,2)$. Следовательно, нормалью к сверхносителю уравнения (4.13) является вектор $N_{1}=(0,1,1)$, а не вектор $\Lambda=(1,3,2)$, указанньй в [60] для оператора $X_{3}$ и являющийся нормалью к сверхносителю решения (4.14). Пошевелим координаты векторов $Q_{i}$ так, чтобы получить векторное произведение, равное $-\Lambda$. Действительно, если вместо $Q_{3}$ взять $Q_{4}=(-3,0,2)$, то

$$
B_{3} \stackrel{\text { def }}{=} Q_{4}-Q_{3}=(-2,0,1)
$$

и векторное произведение $\left[B_{1}, B_{3}\right]=-(1,3,2)$. Следовательно, в уравнении $(4.13)$ имеется опечатка: там пропушен множитель $u$ в третьем слагаемом. Правильное уравнение с автомодельным решением (4.14) есть

$$
u_{t}+u u_{x}+\beta u u_{t t t}=0 .
$$

Действительно, в оригинальной статье [73] рассматривается именно это уравнение под номером (0.4), а уравнение (4.13) это результат опечатки в [60].

3. Страницы 299-300, п. 13.4.1 [60] (см. также [106]). Для уравнения $u_{t x}-\left(u u_{x}\right)_{x}-$ $u_{y y}=0$ указано решение (второе на с. 300) $u^{2}=\left(4 x / t-y^{2} / t^{2}\right) / 8$. Здесь $n=4$, $X=(t, x, y, u)$, носитель уравнения состоит из трех точек $Q_{1}=(-1,-1,0,1), Q_{2}=$ $(0,-2,0,2), Q_{3}=(0,0,-2,1)$. Их разности

$$
\begin{aligned}
& B_{1} \stackrel{\text { def }}{=} Q_{2}-Q_{1}=(1,-1,0,1), \\
& B_{2} \stackrel{\text { def }}{=} Q_{3}-Q_{1}=(1,1,-2,0)
\end{aligned}
$$

линейно независимы. Следовательно, размерность уравнения $d=2$. Носитель решения состоит из трех точек $Q_{4}=(0,0,0,2), Q_{5}=(-1,1,0,0), Q_{6}=(-2,0,2,0)$. Их разности

$$
\begin{aligned}
& B_{3} \stackrel{\text { def }}{=} Q_{5}-Q_{4}=(-1,1,0,-2), \\
& B_{4} \stackrel{\text { def }}{=} Q_{6}-Q_{4}=(-2,0,2,-2)
\end{aligned}
$$

линейно независимы. Следовательно, размерность решения равна двум. При этом $B_{2}=B_{3}-B_{4}$, но вектор $B_{1}$ не выражается линейно через $B_{3}$ и $B_{4}$. Следовательно, свойство (4.1) теоремы 2 не выполнено. Но на этом решении $\left(u u_{x}\right)_{x} \equiv 0$, т.е. не выполнено условие теоремы 2 . Это решение удовлетворяет также уравнению $u_{t x}-u_{y y}=0$. Направляющий вектор сверхносителя этого уравнения $B_{2}$ линейно выражается через векторы $B_{3}$ и $B_{4}$, т.е. свойство (4.1) теоремы 2 выполнено для каждого из двух уравнений размерностей 0 и 1 соответственно. 


\section{§5. Степенное преобразование}

Образуем вектор $\ln X=\left(\ln x_{1}, \ldots, \ln x_{n}\right)$. Пусть $A=\left(a_{i j}\right)$ - квадратная неособая $n$-матрица. Замена координат

$$
\ln Y=A \ln X
$$

назьвается степенным преобразованием [10]-[12], [18]. Обратная замена $\ln X=$ $A^{-1} \ln Y$ также является степенным преобразованием. Напомним, что $A^{*}$ - транспонированная матрища $A$.

Теорема 3. При степенном преобразовании (4.1):

1) сумма (1.1) переходит в сумму $\widetilde{f}(Y)=\sum f_{Q} Y^{\widetilde{Q}}$, где $\widetilde{Q}=A^{*-1} Q$;

2) функция $f(X)$ вида (1.4) переходит в функцию $\widetilde{f}(Y)=f(X)$ вида (1.4), при әтом $\mathbf{S S}(\widetilde{f})=A^{*-1} \mathbf{S S}(f)$;

3) дифференциальный полином (2.3) переходит в отночение двух дифференчиальных полиномов $g(Y) / h(Y)=f(X)$, при әтом носитель $\mathbf{S}(h)$ состоит из одной точки $Q=0$, а носитель $\mathbf{S}(g)=A^{*-1} \mathbf{S}(f)$;

4) нормали к носителямм преобразуются линейно по правилу $\widetilde{N}=A N$.

Для доказательства этой теоремы потребуются вспомогательные утверждения. Пусть $\rho(X)$ - произведение логарифмических производных вида

$$
\frac{\partial^{\left\|L^{\prime}\right\|} \ln x_{n}}{\left(\partial \ln x_{1}\right)^{l_{1}} \cdots\left(\partial \ln x_{n-1}\right)^{l_{n-1}}} \stackrel{\text { def }}{=} \frac{\partial^{\left\|L^{\prime}\right\|} \ln x_{n}}{\left(\partial \ln X^{\prime}\right)^{L^{\prime}}},
$$

где $L^{\prime}=\left(l_{1}, \ldots, l_{n-1}\right), l_{i} \geqslant 0$. Такую производную можно расписать в обычном виде, как дифференциальньй полином. Следовательно, произведение $\rho(X)$ таких производных также является дифференциальньм полиномом. Очевидно, $\mathbf{S}(\rho)=0$. Назовем $\rho(X)$ дифференииально логарифмическим мономом. Сумму

$$
\sum_{i=1}^{s} X^{Q_{i}} \rho_{i}(X)
$$

где $\rho_{i}(X)$ суть дифференциально логарифмические мономы, назовем логарифмичесжой формой. Очевидно, всякую логарифмическую форму (5.3) можно записать в виде дифференциального полинома (2.3). Справедливо и обратное:

ЛЕмма 1. Каждый дифференциальный полином (2.3) можно записать в виде логарифмической формы (5.3).

ДокАЗАТЕЛЬСТво достаточно провести для одного дифференциального монома $a(X)$. Как показано в доказательстве теоремы 2.2 гл. VI [30], справедливо равенство

$$
\frac{\partial^{l} x_{n}}{\partial x_{1}^{l_{1}} \cdots \partial x_{n-1}^{l_{n-1}}}=X^{\prime L^{\prime}} x_{n} \frac{P_{l}\left(\partial \ln x_{n}, \ldots, \partial^{l} \ln x_{n}\right)}{\left(\partial \ln x_{1}\right)^{l_{1}} \cdots\left(\partial \ln x_{n-1}\right)^{l_{n-1}}},
$$


где $l=l_{1}+\cdots+l_{n-1}$ и $P_{l}\left(\xi_{1}, \ldots, \xi_{l}\right)$ - определенные многочлены с постоянными коэффициентами, содержашие только члены вида

$$
\text { const } \xi_{1}^{k_{1}} \cdots \xi_{l}^{k_{l}} \quad \text { c } \quad k_{1}+2 k_{2}+\cdots+l k_{l}=l, \quad k_{i} \geqslant 0
$$

Поскольку дифференциальньй моном $a(X)$ является произведением обычного монома и нескольких производных вида (5.4), то согласно (5.4) он записывается в виде

$$
a(X)=X^{Q} \sum_{i=1}^{t} \rho_{i}(X),
$$

где $Q=Q(a)$ и $\rho_{i}$ суть дифференциально логарифмические мономы. Доказательство окончено.

ЛЕМма 2. Пусть функция $f(X) \stackrel{\text { def }}{=} \sum f_{Q} X^{Q}, Q \in \mathbb{Z}_{+}^{n}$, аналитична в нуле $X=0, f(0)=0 u$

$$
a \stackrel{\text { def }}{=} \partial f /\left.\partial x_{n}\right|_{X=0} \neq 0 .
$$

Тогда уравнение $f(X)=0$ имеет аналитическое решение

$$
x_{n}=\varphi\left(X^{\prime}\right) \stackrel{\text { def }}{=} \sum \varphi_{R^{\prime}} X^{\prime R^{\prime}}
$$

əде $R^{\prime} \in \mathbb{Z}_{+}^{n-1}$. При этом

$$
\varphi_{R^{\prime}}=\beta_{R^{\prime}}\left(\left\{f_{Q}\right\}\right) / a^{2 k-1}
$$

где $\beta_{R^{\prime}}$ суть многочлены степени $2 k-1$ от коэффициентов $f_{Q} c\|Q\| \leqslant k \stackrel{\text { def }}{=}\left\|R^{\prime}\right\|$.

ДокаЗАтЕльство. Первое утверждение см. в [58; $\S 184]$. Докажем второе утверждение. Подставляя разложение (5.5) в разложение для $f(X)$ и приравнивая члены при $X^{\prime R^{\prime}}$, получим равенства

$$
a \varphi_{R^{\prime}}+P_{R^{\prime}}\left(\left\{\varphi_{S^{\prime}}\right\}\right)=0
$$

где $\left\|S^{\prime}\right\| \leqslant\left\|R^{\prime}\right\|$ и $P_{R^{\prime}}$ суть конечные суммы членов вида

$$
f_{Q} \varphi_{R_{1}^{\prime}} \cdots \varphi_{R_{l}^{\prime}}
$$

с $Q \geqslant 0, R_{i} \geqslant 0,\|Q\| \geqslant 2$ и

$$
R_{1}^{\prime}+\cdots+R_{l}^{\prime}=R^{\prime}, \quad l=\|Q\| \geqslant 2 .
$$

Теперь индукцией по $k=\left\|R^{\prime}\right\|$ докажем формулу (5.6). Для $\left\|R^{\prime}\right\|=1$ она, очевидно, следует из (5.7). Пусть целое $k>1$ фиксировано и формула (5.6) верна для $\left\|R^{\prime}\right\|<k$. Докажем ее для $\left\|R^{\prime}\right\|=k$. По индуктивному предположению член (5.8) согласно (5.9) содержит $a^{-1}$ в степени

$$
2 R_{1}^{\prime}-1+\cdots+2 R_{l}^{\prime}-1 \leqslant 2 R^{\prime}-l \leqslant 2 R^{\prime}-2
$$

и является полиномом этой степени от остальных коэффипиентов $f_{P}$. Поэтому согласно (5.7) и (5.8) справедлива формула (5.6) для $\left\|R^{\prime}\right\|=k$. Доказательство окончено. 
ЛЕмМа 3. Пусть

$$
x_{n}=\varphi\left(X^{\prime}\right) \stackrel{\text { def }}{=} \sum \varphi_{Q^{\prime}} X^{\prime Q^{\prime}} \quad \text { no } \quad Q^{\prime} \in \mathbb{Z}_{+}^{n-1}
$$

- локальное разложение с произвольными коэффициентами $\varphi_{Q^{\prime}}$. Пусть в результате аффинного преобразования

$$
X=W Y, \quad \operatorname{det} W \neq 0, \quad W=\left(w_{i j}\right),
$$

из разложения (5.5) получается разложение

$$
y_{n}=\psi\left(Y^{\prime}\right) \stackrel{\text { def }}{=} \sum \psi_{R^{\prime}} Y^{\prime R^{\prime}} \quad \text { no } \quad R^{\prime} \in \mathbb{Z}_{+}^{n-1} .
$$

Тогда его коэффициенты имеют вид

$$
\psi_{R^{\prime}}=\beta_{R^{\prime}}\left(\left\{\varphi_{Q^{\prime}}\right\}\right) / a^{2\left\|R^{\prime}\right\|-1},
$$

где $\beta_{R^{\prime}}\left(\left\{\varphi_{Q^{\prime}}\right\}\right)$ суть полиномы от коэффициентов $\varphi_{Q^{\prime}}$ c $\left\|Q^{\prime}\right\| \leqslant\left\|R^{\prime}\right\| u$

$$
a=w_{n n}-\sum_{i=1}^{n-1} \varphi_{E_{i}} w_{i n},
$$

əде $E_{i}$ обозначает $i$-й единичныц вектор.

ДокАЗАТЕЛЬСтво. Запишем разложение (5.10) в виде $f(X) \stackrel{\text { def }}{=} x_{n}-\varphi\left(X^{\prime}\right)=0$ и сделаем в этом уравнении замену (5.11). Тогда оно примет вид

$$
f(X)=g(Y) \stackrel{\text { def }}{=} \sum_{i=1}^{n} w_{n i} y_{i}-\varphi\left((A Y)^{\prime}\right)=0 .
$$

Рассмотрим его как уравнение для неявной функции (5.12). Из (5.14) видно, что $a \stackrel{\text { def }}{=} \partial g / \partial y_{n}$ в нуле имеет вид (5.13). Применяя к уравнению (5.14) лемму 2 , получим существование и единственность разложения (5.12) и указанный вид его коэффициентов. Доказательство окончено.

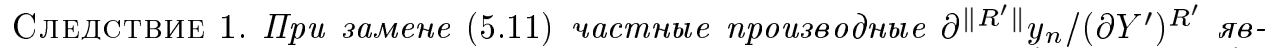

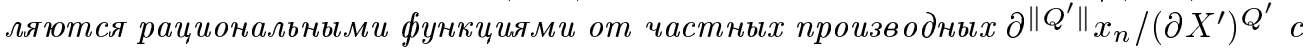
$\left\|Q^{\prime}\right\| \leqslant\left\|R^{\prime}\right\|$, причем знаменатели әтих рациональных функций являются степенями суммы

$$
a \stackrel{\text { def }}{=} w_{n n}-\sum_{i=1}^{n-1} w_{n i} \partial x_{n} / \partial x_{i} .
$$

Действительно, согласно формуле Тейлора

$$
\varphi_{Q^{\prime}}=\frac{1}{Q^{\prime} !} \frac{\partial^{\left\|Q^{\prime}\right\|} x_{n}}{\left(\partial X^{\prime}\right)^{Q^{\prime}}}, \quad \psi_{R^{\prime}}=\frac{1}{R^{\prime} !} \frac{\partial^{\left\|R^{\prime}\right\|} y_{n}}{\left(\partial Y^{\prime}\right)^{R^{\prime}}},
$$

где $Q^{\prime} !=q_{1} ! \cdots q_{n-1}$ !, т.е. производные отличаются от коэффициентов разложений (5.10) и (5.12) постоянными множителями. 
СЛЕДСТвИЕ 2. Пусть $A=W^{-1}=\left(a_{i j}\right)$. Тогда в ситуации леммъ 3 частные производные $\partial^{\left\|Q^{\prime}\right\|} x_{n} /\left(\partial X^{\prime}\right)^{Q^{\prime}}$ являются рациональнымми функциями от производных $\partial^{\left\|R^{\prime}\right\|} y_{n} /\left(\partial Y^{\prime}\right)^{R^{\prime}}$ c $\left\|R^{\prime}\right\| \leqslant\left\|Q^{\prime}\right\|$, причем их знаменатели являются степенями суммы

$$
a_{n n}-\sum_{i=1}^{n-1} a_{i n} \partial y_{n} / \partial y_{i}
$$

ЗАмЕчАниЕ 1. Если матрица $W$ имеет блочнотреугольный вид, указанньй в $\S 2$, гл. VI [30], т.е. $w_{1 n}=\cdots=w_{n-1 n}=0$, то согласно (5.13) $a=w_{n n}=$ const и производные $\partial^{\left\|R^{\prime}\right\|} y_{n} /\left(\partial Y^{\prime}\right)^{R^{\prime}}$ являются полиномами от производных $\partial^{\left\|Q^{\prime}\right\|} x_{n} /\left(\partial X^{\prime}\right)^{Q^{\prime}}$. Справедливо и обратное, т.е. вторые производные являются многочленами от первых, ибо в этом случае $a_{1 n}=\cdots=a_{n-1 n}=0$ и сумма (5.10) равна константе $a_{n n}$.

ДОКАЗАТЕЛЬСТво ТЕОРЕМЫ 3. Утверждение 1 - это свойство 1 степенного преобразования из $\S 3$, гл. II [30].

Утверждение 2 теоремы следует из того, что $X^{B_{i}}=Y^{\widetilde{B}_{i}}$, где $\widetilde{B}_{i}=A^{*-1} B_{i}$.

Докажем утверждение 3 теоремы. Сначала согласно лемме 1 запишем дифференциальный полином в логарифмической форме $(5.3)$, где $\rho_{i}(X)$ суть произведения производных вида (5.2). После преобразования (5.1) $X^{Q_{i}}=Y^{\widetilde{Q}_{i}}$, где $\widetilde{Q}_{i}=A^{*-1} Q_{i}$. Обозначим

$$
\xi_{i}=\ln x_{i}, \quad \eta_{i}=\ln y_{i}, \quad i=1, \ldots, n .
$$

В этих обозначениях производная (5.2) есть

$$
\partial^{\left\|L^{\prime}\right\|} \xi_{n} /\left(\partial \Xi^{\prime}\right)^{L^{\prime}},
$$

апреобразование (5.1) является афффинным преобразованием $\mathrm{H}=A \Xi$. Согласно следствию 2 из леммы 3 производные (5.16) являются рациональньми функциями от производных $\partial^{\left\|M^{\prime}\right\|} \eta_{n} /\left(\partial \mathrm{H}^{\prime}\right)^{M^{\prime}}$ с $\left\|M^{\prime}\right\| \leqslant\left\|L^{\prime}\right\|$, причем знаменателями служат степени суммы

$$
\widetilde{a}=a_{n n}-\sum_{i=1}^{n-1} a_{i n} \partial \eta_{n} / \partial \eta_{i} .
$$

Следовательно, дифференциально логарифмический моном $\rho_{i}(X)$ является отношением $\widetilde{\rho}_{i}(Y) / \widetilde{a}^{m}$, где $\widetilde{\rho}_{i}$ - полином от производных вида $\partial^{\left\|M^{\prime}\right\|} \ln y_{n} /\left(\partial \ln Y^{\prime}\right)^{M^{\prime}}$ и $\widetilde{a}=$ $a_{n n}-\sum_{i=1}^{n-1} a_{i n} \partial \ln y_{n} / \partial \ln y_{i}$, а вся сумма (5.3) есть $g(Y) / \widetilde{a}^{\tilde{m}}$, где $g(Y)$ - логарифмическая форма некоторого дифференциального полинома и целое $\widetilde{m} \geqslant 0$. Поскольку носители дифференциальных полиномов $\widetilde{\rho}_{i}(Y)$ и $\widetilde{a}(Y)$ состоят из одной точки - нуля, то носитель $\mathbf{S}\left(\widetilde{a}^{\widetilde{m}}\right)=0$ и носитель $\mathbf{S}(g)=A^{*-1} \mathbf{S}(f)$. Это завершает доказательство третьего утверждения теоремы 3.

Четвертое утверждение следует из первых трех, ибо для сохранения скалярного произведения преобразование (5.1) индуцирует в сопряженном пространстве $\mathbb{R}_{*}^{n}$ преобразование $\widetilde{N}=A N$. Доказательство теоремы 3 окончено.

Умножению дифференциального полинома $f(X)$ на моном $X^{T}$ соответствует в $\mathbb{R}^{n}$ параллельный перенос носителей $\mathbf{S}(f)$ и $\mathbf{S} \mathbf{S}(f)$ на вектор $T$. Поэтому если 
$d(f)=d<n$, то линейным преобразованием вида $\widetilde{Q}=A^{*-1}(Q+T)$ можно поместить множество $\mathbf{S}(f)$ в координатное подпространство

$$
\widetilde{q}_{1}=\cdots=\widetilde{q}_{n-d}=0 .
$$

TЕорема 4. Для дифференциального полинома $f(X)$ с $d(f) \stackrel{\text { def }}{=} d<n$ существуют вектор $T \in \mathbb{R}^{n}$ и матрица А такие, что степенное преобразование (5.1) приводит дифференциальное уравнение $X^{T} f(X)=0 \kappa$ виду $g(Y)=0$, где для всех точек $\widetilde{Q}$ носителя $\mathbf{S}(g)$ выполнено свойство (5.17).

ДокАЗАТЕЛЬСТВо следует из утверждения 3 теоремы 3 и решения задачи 3 в $\S 11$ гл. I [30]. В качестве вектора $-T$ можно взять любой вектор из сверхносителя $\mathbf{S S}(f)$. Доказательство окончено.

ЗАМЕчАниЕ 2. Если координата $x_{i}$ присутствует в полиноме (2.3) только под знаком дифференциала, то можно также делать степенные замены вида $d x_{i}=Y^{B_{i}} d y_{i}$, где $B_{i}=\left(b_{i 1}, \ldots, b_{i n}\right)$ и $b_{i i}=0$.

ПримеР 7 (продолжение примеров 2-4). Для уравнения (2.4) $n=3$ и $d=1$. В обозначениях (2.5) сделаем степенное преобразование (5.1)

$$
\begin{aligned}
& y_{1}=x_{1}, \\
& y_{2}=x_{2}, \\
& y_{3}=X^{B / \sigma}=x_{1}^{1 / \sigma} x_{2}^{-2 / \sigma} x_{3}
\end{aligned}
$$

с матрищей

$$
A=\left(\begin{array}{ccc}
1 & 0 & 0 \\
0 & 1 & 0 \\
1 / \sigma & -2 / \sigma & 1
\end{array}\right)
$$

В исходных координатах $t, x, u$ это преобразование $u=v t^{-1 / \sigma} x^{2 / \sigma}$. После сокращения на $t^{-1-1 / \sigma} x^{2 / \sigma}$ уравнение (2.4) переходит в уравнение

$$
\left(v_{t} t\right)-v / \sigma=\left(v_{x x} x^{2}\right) v^{\sigma}+\sigma\left(v_{x} x\right)^{2} v^{\sigma-1}+4(\sigma+1) \sigma^{-1}\left(v_{x} x\right) v^{\sigma}+2(2+\sigma) \sigma^{-2} v^{\sigma+1} .
$$

Носитель этого уравнения состоит из двух точек

$$
(0,0,1) \text { и }(0,0,1+\sigma) .
$$

Здесь вектор $T=(1+1 / \sigma,-2 / \sigma, 0)$ и матрица $A$ дается формулой (5.18). В качестве $x_{1}, x_{2}$ вместо $t$ и $x$ можно взять $w_{1}$ и $w_{2}$. 


\section{§6. Логарифмическое преобразование}

Пусть у всех точек $\widetilde{Q}$ носителя дифференциального полинома $g(Y)$ координата $\widetilde{q}_{i}=0$. Тогда координата $y_{i}$ входит в $g(Y)$ только в виде степеней дифференшиала $\partial \ln y_{i}$. Поэтому если сделать логарифмическое преобразование $z_{i}=\ln y_{i}$, то $g(Y)$ станет дифференциальным полиномом от $y_{1}, \ldots, y_{i-1}, z_{i}, y_{i+1}, \ldots, y_{n}[26]-[31]$.

ТЕОРема 5. Если для всех точек $\widetilde{Q}$ носителя $\mathbf{S}(g)$ дифференииального полинома $g(Y)$ выполнено свойство (5.17), то после логарифмического преобразования

$$
\begin{aligned}
& z_{i}=\ln y_{i}, \quad i=1, \ldots, n-d, \\
& z_{j}=y_{j}, \quad j=n-d+1, \ldots, n,
\end{aligned}
$$

получается дифференииальньй полином $\widetilde{g}(Z)=g(Y)$.

ДокАЗАТЕЛЬСТво. Согласно лемме 1 запишем дифференциальньй полином $g(Y)$ в логарифмической форме

$$
g(Y)=\sum_{k=1}^{t} Y^{\widetilde{Q}_{k}} \widetilde{\rho}_{k}(Y)
$$

где $\widetilde{\rho}_{k}(Y)$ суть дифференциально логарифмические мономы. По условию для всех векторных показателей $\widetilde{Q}_{i}$ вьполнено свойство (5.17), т.е. $y_{1}, \ldots, y_{n-d}$ отсутствуют в мономах $Y^{\widetilde{Q}_{k}}$, имеюшихся в (6.2). Эти координаты имеются только в $\widetilde{\rho}_{k}(Y)$ в виде $\ln y_{i}$. Поэтому после логарифмической замены (6.1) в логарифмической форме (6.2) не возникают $e^{z_{i}}$, т.е. форма (6.2) остается дифференциальным полиномом. Доказательство окончено.

ПримеР 8 (продолжение примера 7). Положим $z_{1}=\ln t, z_{2}=\ln x$. Поскольку $v_{t} t=\partial v / \partial z_{1}, v_{x} x=\partial v / \partial z_{2}, v_{x x} x^{2}=\partial^{2} v / \partial z_{2}^{2}-\partial v / \partial z_{2}$, то уравнение (5.14) принимает вид

$$
\frac{\partial v}{\partial z_{1}}-\frac{v}{\sigma}=\frac{\partial^{2} v}{\partial z_{2}^{2}} v^{\sigma}+\sigma\left(\frac{\partial v}{\partial z_{2}}\right)^{2} v^{\sigma-1}+\frac{3 \sigma+4}{\sigma} \frac{\partial v}{\partial z_{2}} v^{\sigma}+\frac{2(2+\sigma)}{\sigma^{2}} v^{\sigma+1}
$$

Носитель этого уравнения состоит из точек

$$
(-1,0,1),(0,0,1),(0,-2,1+\sigma),(0,-1,1+\sigma),(0,0,1+\sigma)
$$

Следовательно, две точки (5.20) носителя уравнения (5.19) раздулись в пять точек (6.4) носителя уравнения (6.3).

ПримеР 9 (продолжение примера 5). Рассмотрим уравнение (4.5) сначала в случае (4.7). Сделаем степенное преобразование

$$
\begin{aligned}
& t=t, \\
& y=t^{\kappa} x, \\
& v=X^{B_{2} /(\beta-1)}=t^{1 /(\beta-1)} u,
\end{aligned}
$$


где число $\kappa$ определено в (4.8). При этом векторы $(\kappa, 1,0)$ и $(1 /(\beta-1), 0,1)$ образуют базис линейного подпространства в $\mathbb{R}^{3}$, параллельного сверхносителю уравнения (4.5). Обратное к (6.5) преобразование есть

$$
\begin{aligned}
t & =t, \\
x & =t^{-\kappa} y, \\
u & =t^{1 /(1-\beta)} v .
\end{aligned}
$$

Вычисляем производные, используя (6.5) и (6.6):

$$
\begin{gathered}
u_{t}=\frac{1}{1-\beta} t^{1 /(1-\beta)-1} v+t^{1 /(1-\beta)}\left(v_{t}+v_{y} \kappa t^{-1} y\right), \quad u_{x}=t^{1 /(1-\beta)} v_{y} t^{\kappa}, \\
u^{\sigma} u_{x}=t^{\sigma(1-\beta)+1 /(1-\beta)+\kappa} v^{\sigma} v_{y}=t^{(\sigma+1+\beta) /(2(1-\beta))} v^{\sigma} v_{y} \\
\left(u^{\sigma} u_{x}\right)_{x}=t^{(\sigma+1+\beta) /(2(1-\beta))+\kappa}\left(\sigma v^{\sigma-1} v_{y}^{2}+v^{\sigma} v_{y y}\right)=t^{\beta /(1-\beta)}\left(\sigma v^{\sigma-1} v_{y}^{2}+v^{\sigma} v_{y y}\right) .
\end{gathered}
$$

Подставляя эти значения в уравнение $(4.5)$ и сокращая на $t^{\beta /(1-\beta)}$, получаем уравнение

$$
\frac{1}{1-\beta} v+t v_{t}+\kappa y v_{y}=\sigma v^{\sigma-1} v_{y}^{2}+v^{\sigma} v_{y y}+v^{\beta}
$$

Теперь сделаем логарифмическое преобразование $\tau=\ln t$ (т.е. $t=e^{\tau}$ ). Уравнение (6.7) перейдет в

$$
\frac{1}{1-\beta} v+v_{\tau}+\kappa y v_{y}=\sigma v^{\sigma-1} v_{y}^{2}+v^{\sigma} v_{y y}+v^{\beta}
$$

Если $v$ не зависит от $\tau$, т.е. $v_{\tau}=0$, то это уравнение становится уравнением (4.9). Однако, теперь можно искать решение полного уравнения (4.5) или (6.8) в виде ряда по отрицательньм степеням $\tau$ :

$$
v=\psi(y)+\tau^{-1} \psi_{1}(y)+\tau^{-2} \psi_{2}(y)+\cdots,
$$

или в виде полинома от $\tau$ :

$$
v=\psi(y) \tau^{m}+\psi_{1}(y) \tau^{m-1}+\cdots+\psi_{m}(y)
$$

где $\psi(\xi)$ - решение уравнения (4.9). Это приводит к такому разложению решений $u(t, x)$ уравнения $(4.5)$ по степеням $(\ln t)^{-1}$, которое начинается с автомодельного решения.

Рассмотрим теперь уравнение (4.5) в случае (4.10). Сделаем степенное преобразование

$$
\begin{aligned}
t & =t, \\
x & =x, \\
v & =x^{-2 / \sigma} u,
\end{aligned}
$$


где векторы $(1,0,0)$ и $(0,-2 / \sigma, 1)$ образуют базис двумерного линейного подпространства, параллельного сверхносителю уравнения (4.5). Здесь $u=x^{2 / \sigma} v$. Вычисляем производные:

$$
\begin{gathered}
u_{x}=\frac{2}{\sigma} x^{2 / \sigma-1} v+x^{2 / \sigma} v_{x}, \quad u_{x} u^{\sigma}=\frac{2}{\sigma} x^{2 / \sigma+1} v^{\sigma+1}+x^{2 / \sigma+2} v^{\sigma} v_{x} \\
\left(u_{x} u^{\sigma}\right)_{x}=\frac{2(2+\sigma)}{\sigma^{2}} x^{2 / \sigma} v^{\sigma+1}+\frac{4(\sigma+1)}{\sigma} x^{2 / \sigma+1} v^{\sigma} v_{x}+x^{2 / \sigma+2} v^{\sigma-1} v_{x}^{2}+x^{2 / \sigma+2} v^{\sigma} v_{x x}
\end{gathered}
$$

Подставляя это выражение в уравнение (4.5) и сокращая на $x^{2 / \sigma}$, получаем уравнение

$$
v_{t}=\frac{2(2+\sigma)}{\sigma^{2}} v^{\sigma+1}+\frac{4(\sigma+1)}{\sigma} x v^{\sigma} v_{x}+x^{2} v^{\sigma-1} v_{x}^{2}+x^{2} v^{\sigma} v_{x x}
$$

После логарифмического преобразования $\ln x=\eta$ (т.е. $x=e^{\eta}$ ) это уравнение принимает вид

$$
v_{t}=\frac{2(2+\sigma)}{\sigma^{2}} v^{\sigma+1}+\frac{3 \sigma+4}{\sigma} v^{\sigma} v_{\eta}+v^{\sigma-1} v_{\eta}^{2}+v^{\sigma} v_{\eta \eta}+v
$$

ибо $v_{x}=v_{\eta} / x, v_{x x}=\left(v_{\eta \eta}-v_{\eta}\right) / x^{2}$. Если $v$ не зависит от $\eta$, то последнее уравнение совпадает с уравнением (4.12). Решение уравнения (6.10) можно искать в виде полинома от $\eta$ или ряда по $\eta^{-1}$; при этом коэффициент ведущего члена - это решение уравнения (4.12). В степенных преобразованиях (6.5) и (6.9) и далее вместо $t$ и $x$ можно использовать $w_{1}$ и $w_{2}$.

\section{$\S 7$. Обыкновенное дифференциальное уравнение}

При $n=2$ производная (2.1) является обыкновенной и дифференциальное уравнение $f(X)=0$, соответствующее полиному (2.3), также является обыкновенным. Для него теоремы 1-5 остаются справедливыми. При этом неравенство $d<n$ означает, что $d=0$ или $d=1$. Однако для обыкновенных дифференциальных уравнений можно доказать больше.

Теорема 6. Пусть $n=2$, обыкновенное дифференциальное уравнение $f(X)=0$ имеет порядок $m$ и размерность $d(f)<n$. Тогда посредством степенных $и$ логарифмических преобразований это уравнение можно свести к уравнению порядка $m-(n-d(f))$.

ДокаЗАТЕльство. Пусть сначала $d=1$. Согласно теореме 3 сделаем степенное преобразование, которое переведет сверхноситель $\mathbf{S S}(f)$ в горизонтальную прямую $\widetilde{q}_{2}=$ const $=c$. После сокрашения на $y_{2}^{c}$ получим уравнение $g(Y)=0$, носитель которого лежит на оси $\widetilde{q}_{2}=0$. Его порядок равен $m$. Это уравнение содержит $y_{2}$ только в виде $d \ln y_{2} / d y_{1}$. Беря эту производную в качестве новой зависимой переменной, получаем уравнение порядка $m-1$.

Рассмотрим теперь случай $d(f)=0$, когда носитель $\mathbf{S}(f)$ состоит из одной точки $Q$. Умножая уравнение на $X^{-Q}$, получим уравнение с нулевым носителем. Поскольку $q_{2}=0$, то $x_{2}$ входит в него только в виде $d \ln x_{2} / d x_{1}$. Беря эту производную в качестве новой зависимой переменной, получим уравнение порядка $m-1$. Его носитель 
расположен на вертикальной оси $q_{1}=0$. Поменяв местами зависимую и независимую переменные, что соответствует степенному преобразованию (5.1) с матрищей

$$
A=\left(\begin{array}{ll}
0 & 1 \\
1 & 0
\end{array}\right),
$$

получим уравнение порядка $m-1$, носитель которого расположен на горизонтальной оси $\widetilde{q}_{2}=0$. Повторяя прием, примененный выше, понизим порядок уравнения еще на единицу. Доказательство окончено.

ПРимеР 10. Рассмотрим уравнение Блазиуса, встречаюшееся в теории пограничного слоя [96],

$$
y^{\prime \prime \prime}+y^{\prime \prime} y=0
$$

где штрих означает производную по $x$. Здесь $n=2, m=3, x_{1}=x, x_{2}=y$. Носитель уравнения (7.1) состоит из двух точек $Q_{1}=(-3,1)$ и $Q_{2}=(-2,2)$, лежаших на прямой $q_{1}-q_{2}+4=0$, т.е. $d=1$. Следовательно, $m-(n-d)=2$. Согласно замечанию $2 \S 5$ сделаем степенное преобразование $d t=y d x$. Тогда $d t / d x=y$. Будем точкой обозначать дифференцирование по $t$. Имеем

$$
y^{\prime}=\dot{y} y, \quad y^{\prime \prime}=\ddot{y} y^{2}+\dot{y}^{2} y, \quad y^{\prime \prime \prime}=\dddot{y} y^{3}+4 \ddot{y} \dot{y} y^{2}+\dot{y}^{3} y .
$$

Подставляя производные в уравнение (7.1) и сокрашая на $y^{4}$, получаем уравнение

$$
\dddot{y} y^{-1}+4 \ddot{y} \dot{y} y^{-2}+\dot{y}^{3} y^{-3}+\dot{y} y^{-1}+\dot{y}^{2} y^{-2}=0 \text {. }
$$

Сделаем в нем логарифмическое преобразование $\ln y=z, y=e^{z}$. Тогда

$$
\dot{y}=\dot{z} y, \quad \ddot{y}=\ddot{z} y+\dot{z}^{2} y, \quad \dddot{y}=\dddot{z} y+3 \ddot{z} \dot{z} y+\dot{z}^{3} y .
$$

Подставляя эти производные в уравнение (7.2) и приводя подобные члены, получаем уравнение

$$
\dddot{z}+7 \ddot{z} \dot{z}+6 \dot{z}^{3}+\ddot{z}+2 \dot{z}^{2}=0 .
$$

Полагая здесь $\dot{z}=u$, получаем уравнение второго порядка

$$
\ddot{u}+7 \dot{u} u+6 u^{3}+\dot{u}+2 u^{2}=0 .
$$

Носитель этого уравнения состоит из пяти точек, а его размерность равна двум.

Если размерность $d(f)<n$, то уравнение $f(X)=0$ и его решения являются квазиоднородньми. Поэтому одну граничную задачу на двух конщах интервала можно сводить к двум граничным задачам на одном конце интервала (см. [60; гл. 16], [70], [71], [82]). В следующих двух теоремах предполагается, что решение любой граничной задачи $f(X)=0, x_{2}^{(k)}(0)=\alpha_{k}, k=0, \ldots, m-1$, сушествует и единственно. 
Теорема 7. Пусть $f(X)=0$ - обькновенное дифференциальное уравнение порядка т, размерности 1 и вектор $\Lambda=\left(\lambda_{1}, \lambda_{2}\right)$ нормален $\kappa$ его сверхносителю. Если $l \lambda_{1} \neq \lambda_{2}$, то решение граничной задачи

$$
\begin{gathered}
x_{2}(0)=x_{2}^{\prime}(0)=\cdots=x_{2}^{(m-2)}(0)=0, \\
x_{2}^{(l)}(\infty)=c \neq 0
\end{gathered}
$$

сводится к решению двух граничных задач с условиями только в нуле $x_{1}=0$.

ДокАЗАТЕльство. Пусть $x_{2}=\varphi\left(x_{1}\right)$ - решение граничной задачи (7.3), $x_{2}^{(m-1)}(0)=1$. Пусть $\varphi^{(l)}(\infty) \stackrel{\text { def }}{=} \gamma \neq 0, \infty$. При подстановке (3.2) уравнение переходит в себя, а решение $x_{2}=\varphi\left(x_{1}\right)$ переходит в решение

$$
x_{2}=\mu^{-\lambda_{2}} \varphi\left(\mu^{\lambda_{1}} x_{1}\right) .
$$

Его $k$-я производная есть

$$
x_{2}^{(k)}=\mu^{-\lambda_{2}+k \lambda_{1}} \varphi^{(k)}\left(\mu^{\lambda_{1}} x_{1}\right) .
$$

Решение (7.5), очевидно, удовлетворяет нулевьм граничньм условиям (7.3). Оно удовлетворяет граничному условию (7.4), если $\mu^{-\lambda_{2}+l \lambda_{1}} \gamma=c$, т.е. $\mu=\mu_{0} \stackrel{\text { def }}{=}$ $(c / \gamma)^{1 /\left(l \lambda_{1}-\lambda_{2}\right)}$, ибо по условию теоремы $-\lambda_{2}+l \lambda_{1} \neq 0$. При этом согласно (7.6) на решении (7.5) с $\mu=\mu_{0}$ имеем

$$
x_{2}^{(m-1)}(0)=\mu_{0}^{-\lambda_{2}+(m-1) \lambda_{1}} \varphi^{(m-1)}(0)=(c / \gamma)^{\left[(m-1) \lambda_{1}-\lambda_{2}\right] /\left(l \lambda_{1}-\lambda_{2}\right)} .
$$

Поэтому, чтобы решить граничную задачу $(7.3),(7.4)$, надо сначала решить граничную задачу $(7.3), x_{2}^{(m-1)}(0)=1$ и найти значение $\gamma \stackrel{\text { def }}{=} \varphi^{(l)}(\infty)$, а затем решить вторую граничную задачу (7.3), (7.7). Доказательство окончено.

Если размерность уравнения равна нулю, то уравнение инвариантно относительно замен (3.2) с двумя независимыми параметрами. Поэтому указанная в теореме 7 редукция возможна и в том случае, когда при $x_{1}=0$ имеется одно ненулевое граничное условие. Для уравнения второго порядка эта ситуация разобрана в [60; $§ 16.1]$, [70], [71].

Теорема 8. Пусть $f(X)=0$ - обыкновенное дифференциальное уравнение порядка $m \geqslant 2$ и размерности 0. В случае общего положсения решение граничной задачи

$$
\begin{gathered}
x_{2}(0)=x_{2}^{\prime}(0)=\cdots=x_{2}^{(m-3)}(0)=0, \\
x_{2}^{(m-2)}(\infty)=a, \quad x_{2}^{(m-1)}(\infty)=b
\end{gathered}
$$

сводится к решению двух граничньх задач с условиями только в нуле $x_{1}=0$. 
ДокАЗАТЕЛЬСтво. Пусть $x_{2}=\varphi\left(x_{1}\right)$ - решение граничной задачи $(7.8)$,

$$
x_{2}^{(m-2)}(0)=x_{2}^{(m-1)}(0)=1
$$

и $\varphi^{(m-2)}(\infty)=\alpha \neq 0, \varphi^{(m-1)}(\infty)=\beta \neq 0, \infty$. Поскольку уравнение $f(X)=0$ нульмерно, оно инвариантно при заменах $\widetilde{x}_{1}=\mu x_{1}, \widetilde{x}_{2}=x_{2}$ и $\widetilde{x}_{1}=x_{1}, \widetilde{x}_{2}=\nu x_{2}$, где $\mu, \nu \in \mathbb{R}, \mu, \nu>0$. Поэтому при любых $\mu, \nu>0$ выражение $x_{2}=\nu \varphi\left(\mu x_{1}\right)$ также является решением уравнения $f(X)=0$. Оно, очевидно, удовлетворяет граничным условиям (7.8). Поскольку для этого решения

$$
x_{2}^{(k)}=\nu \mu^{k} \varphi^{(k)}\left(\mu x_{1}\right),
$$

то оно удовлетворяет условиям (7.9), если

$$
\nu \mu^{m-2} \alpha=a, \quad \nu \mu^{m-1} \beta=b .
$$

Из этих уравнений определяются конкретные значения параметров $\mu=\mu_{0}, \nu=\nu_{0}$, которые им удовлетворяют: $\mu_{0}=(b / \beta)(a / \alpha)^{-1}, \nu_{0}=(a / \alpha) \mu_{0}^{2-m}=(a / \alpha)^{m-1}(b / \beta)^{2-m}$. При $x_{1}=0$ это решение $x_{2}=\nu_{0} \varphi\left(\mu_{0} x_{1}\right)$ имеет

$$
x_{2}^{(m-2)}(0)=\nu_{0} \mu_{0}^{m-2}=a / \alpha, \quad x_{2}^{(m-1)}(0)=\nu_{0} \mu_{0}^{m-1}=b / \beta .
$$

Поэтому для решения задачи (7.8), (7.9) надо сначала решить задачу $(7.8),(7.10)$ и найти значения $\alpha, \beta$, а затем решить задачу (7.8), (7.11). Доказательство окончено.

Частные случаи теорем 7 и 8 имеются в [71], [82], там же рассмотрены другие виды граничных условий и конечньй интервал изменения независимой переменной. Там же имеются примеры применения теорем 7 и 8.

\section{$\S 8$. Система уравнений}

Пусть $X^{\prime}=\left(x_{1}, \ldots, x_{n^{\prime}}\right)$ - независимые переменные, $X^{\prime \prime}=\left(x_{n^{\prime}+1}, \ldots, x_{n}\right)$ - зависимые переменные. Аналогично каждый вектор $Y$ длины $n$ будем разбивать на два подвектора $Y^{\prime}$ и $Y^{\prime \prime}$ длины $n^{\prime}$ и $n-n^{\prime}$ соответственно. Дифференциальны.м мономом $a(X)$ назовем произведение степеней $X$ и производных вида

$$
\partial^{\left\|L^{\prime}\right\|} x_{j} / \partial X^{\prime L^{\prime}}
$$

где $L^{\prime} \in \mathbb{Z}_{+}^{n^{\prime}}$ и $j>n^{\prime}$. Каждому дифференциальному моному $a(X)$ поставим в соответствие точку $Q(a) \in \mathbb{R}^{n}$ (его векторный показатель степени) по следующему правилу: моному const $X^{Q}$ соответствует точка $Q$; производной (8.1) соответствует точка

$$
Q=\left(-L^{\prime}, E_{j}\right), \text { т.е. } Q^{\prime}=-L^{\prime}, \quad Q^{\prime \prime}=E_{j} ;
$$

а произведению двух дифференциальных мономов $a(X)$ и $b(X)$ соответствует точка $Q(a b)=Q(a)+Q(b)$. Конечная сумма дифференциальных мономов

$$
f(X)=\sum_{i=1}^{s} a_{i}(X)
$$


назьвается дифференииальным полиномом. Множество точек $\mathbf{S}(f) \stackrel{\text { def }}{=}\left\{Q\left(a_{i}\right), i=\right.$ $1, \ldots, s\}$ назьвается носителем дифференциального полинома (8.3). Аффинная оболочка $\mathbf{S S}(f)$ носителя $\mathbf{S}(f)$ назьвается сверхносителем полинома (8.3). Аналогично определяются его размерность $d(f) \stackrel{\text { def }}{=} \operatorname{dim} \mathbf{S S}(f)$ и нормальное подпространство $\mathbf{N}(f) \stackrel{\text { def }}{=} \mathbf{N}(\mathbf{S S}(f)) \subset \mathbb{R}_{*}^{n}$.

Рассмотрим совокупность дифференциальных полиномов

$$
f_{1}(X), \ldots, f_{m}(X)
$$

Каждый из полиномов $f_{i}(X)$ имеет свои носитель $\mathbf{S}\left(f_{i}\right)$, нормальное подпространство $\mathbf{N}\left(f_{i}\right)$ и размерность $d\left(f_{i}\right)$. Однако нормальным подпространством $\mathbf{N}$ совокупносmu (8.4) является пересечение

$$
\mathbf{N}=\mathbf{N}\left(f_{1}\right) \cap \cdots \cap \mathbf{N}\left(f_{m}\right),
$$

а его коразмерность $d$, т.е. $d=n-\operatorname{dim} \mathbf{N}$, является размерностью совокупнос$m u$ (8.4). Если совокупности (8.4) поставить в соответствие систему уравнений

$$
f_{i}(X)=0, \quad i=1, \ldots, m,
$$

то аналоги теорем 1-8 справедливы и для совокупности (8.4), и для системы (8.6). Приведем их формулировки без доказательств, ибо их доказательства почти дословно повторяют доказательства соответствующих теорем 1-8.

Система уравнений (8.6) допускает оператор Ли (3.1), если каждое из уравнений инвариантно относительно замены (3.2).

Теорема 9. Система уравнений (8.6) допускает оператор Ли (3.1), если вектор $\Lambda=\left(\lambda_{1}, \ldots, \lambda_{n}\right)$ лежит в пересечении (8.5).

Выражение $X^{\prime \prime}=\Phi^{\prime \prime}\left(X^{\prime}\right)$ называется решением системы уравнений (8.6), если при этой подстановке каждое уравнение обрашается в тождество по $X^{\prime}$. Решение является автомодельным, если оно допускает оператор вида (3.1), т.е. инвариантно относительно замен (3.2). Если решение $X^{\prime \prime}=\Phi^{\prime \prime}\left(X^{\prime}\right)$ допускает оператор (3.1), то все сверхносители $\mathbf{S S}\left(x_{j}-\varphi_{j}\left(X^{\prime}\right)\right), j=n^{\prime}+1, \ldots, n$, нормальны к вектору $\Lambda$. При этом сверхноситель $\mathbf{S S}\left(x_{j}-\varphi_{j}\left(X^{\prime}\right)\right)$ проходит через точку $E_{j}$, а сверхноситель $\mathbf{S S}\left(\varphi_{j}\left(X^{\prime}\right)\right)$ расположен в координатном подпространстве $Q^{\prime \prime}=0$. Поэтому сверхноситель $\mathbf{S S}\left(\varphi_{j}\left(X^{\prime}\right)\right)$ расположен в линейном многообразии

$$
\left\langle\Lambda^{\prime}, Q^{\prime}\right\rangle=\lambda_{j}, \quad Q^{\prime \prime}=0
$$

ТЕОрема 10. Пусть $X^{\prime \prime}=\Phi^{\prime \prime}\left(X^{\prime}\right)$ - такое решение системы (8.6), которое не удовлетворяет никакой системе $\breve{f}_{i}=0, i=1, \ldots, m$, образованной собственньми подсуммами суммы $f_{i}$ и имеющей размерность, меньшую, чем размерность системы (8.6). Тогда нормальное подпространство решения лехсит в нормальном подпространстве системы. 
ПРимеР 11. Рассмотрим систему (1.6) из [72; с. 102]

$$
\begin{aligned}
& u_{t}=\left(u^{\sigma_{1}} u_{x}\right)_{x}+u^{\beta_{1}} v^{\gamma_{2}}, \\
& v_{t}=\left(u^{\sigma_{2}} v_{x}\right)_{x}+v^{\beta_{2}} u^{\gamma_{1}}, \quad \beta_{i}, \gamma_{i} \geqslant 0, \quad \sigma_{i}>0 .
\end{aligned}
$$

Функции $u, v$ могут быть температурами или концентрациями двух веществ в автокаталитической реакции. Здесь $X=(t, x, u, v), n=4, n^{\prime}=2$. Носитель первого уравнения состоит из трех точек $Q_{1}=(-1,0,1,0), Q_{2}=\left(0,-2, \sigma_{1}+1,0\right), Q_{3}=$ $\left(0,0, \beta_{1}, \gamma_{2}\right)$. Носитель второго уравнения состоит из трех точек $Q_{4}=(-1,0,0,1)$, $Q_{5}=\left(0,-2,0, \sigma_{2}+1\right), Q_{6}=\left(0,0, \gamma_{1}, \beta_{2}\right)$. Составим разности

$$
\begin{aligned}
& B_{1} \stackrel{\text { def }}{=} Q_{2}-Q_{1}=\left(1,-2, \sigma_{1}, 0\right), \\
& B_{2} \stackrel{\text { def }}{=} Q_{3}-Q_{1}=\left(1,0, \beta_{1}-1, \gamma_{2}\right), \\
& B_{3} \stackrel{\text { def }}{=} Q_{5}-Q_{4}=\left(1,-2,0, \sigma_{2}\right), \\
& B_{4} \stackrel{\text { def }}{=} Q_{6}-Q_{4}=\left(1,0, \gamma_{1}, \beta_{2}-1\right) .
\end{aligned}
$$

Нормальное подпространство $\mathbf{N}$ системы (8.8) нормально к четырем векторам $B_{1}, B_{2}$, $B_{3}, B_{4}$. Его размерность больше нуля, только если $\Delta \stackrel{\text { def }}{=} \operatorname{det}\left(B_{1} B_{2} B_{3} B_{4}\right)^{*}=0$. Вычисления дают

$$
\Delta=-2\left[\sigma_{1}\left(\beta_{2}-1-\gamma_{2}\right)+\sigma_{2}\left(\gamma_{1}+1-\beta_{1}\right)\right] .
$$

Чтобы упростить вычисления определителя по (8.9), удобно из третьей строчки вычесть первую, а из четвертой - вторую. Следовательно, $\mathbf{N} \ni \Lambda \neq 0$, только если $\Delta=0$, т.е.

$$
\frac{\sigma_{1}}{\sigma_{2}}=\frac{\gamma_{1}+1-\beta_{1}}{\gamma_{2}+1-\beta_{2}} .
$$

Вычисляя алгебраические дополнения размера $3 \times 3$ в матрице $\left(B_{1} B_{2} B_{3}\right)^{*}$, получаем вектор нормали

$$
\Lambda=\left(\lambda_{1}, \lambda_{2}, \lambda_{3}, \lambda_{4}\right)=\left(-2\left(\sigma_{2}\left(\beta_{1}-1\right)+\sigma_{1} \gamma_{2}\right), \sigma_{1} \sigma_{2}-\sigma_{1} \gamma_{2}-\sigma_{2}\left(\beta_{1}-1\right), 2 \sigma_{2}, 2 \sigma_{1}\right) .
$$

Согласно (8.7) для автомодельного решения $u=\varphi(t, x), v=\psi(t, x)$ сверхноситель функции $\varphi$ расположен на прямой $\lambda_{1} q_{1}+\lambda_{2} q_{2}=\lambda_{3}, q_{3}=q_{4}=0$, а сверхноситель функции $\psi$ расположен на прямой $\lambda_{1} q_{1}+\lambda_{2} q_{2}=\lambda_{4}, q_{3}=q_{4}=0$. Следовательно,

$$
u=t^{m_{1}} g_{1}(\xi), \quad v=t^{m_{2}} g_{2}(\xi)
$$

где $m_{1}=\lambda_{3} / \lambda_{1}, m_{2}=\lambda_{4} / \lambda_{1}, \xi=t^{\kappa} x, \kappa=-\lambda_{2} / \lambda_{1}$. При этом функции $g_{1}$ и $g_{2}$ удовлетворяют системе обыкновенных дифференциальных уравнений

$$
\begin{aligned}
& m_{1} g_{1}+\kappa \xi g_{1}^{\prime}=\left(g_{1}^{\sigma_{1}} g_{1}^{\prime}\right)^{\prime}+g_{1}^{\beta_{1}} g_{2}^{\gamma_{2}}, \\
& m_{2} g_{2}+\kappa \xi g_{2}^{\prime}=\left(g_{2}^{\sigma_{2}} g_{2}^{\prime}\right)^{\prime}+g_{2}^{\beta_{2}} g_{1}^{\gamma_{1}},
\end{aligned}
$$

где штрих означает дифференцирование по $\xi$. В формуле (8.10) и далее вместо $t$ и $x$ можно использовать $w_{1}=c_{1}+\delta_{1} t$ и $w_{2}=c_{2}+\delta_{2} x$. 
ТЕОРема 11. При степенном преобразовании (5.1) дифференциальный полином (8.3) переходит в отношение двух дифференциальных полиномов $g(Y) / h(Y)=f(X)$, при этом носитель $\mathbf{S}(h)$ состоит из одной точки $Q=0$, а носитель $\mathbf{S}(g)=A^{*-1} \mathbf{S}(f)$. Нормали $\kappa$ носителям преобразуются по правилу $\widetilde{N}=A N$.

ТЕОРема 12. Для совокупности дифференииальных полиномов (8.4) с размерностью $d<n$ существуют векторы $T_{1}, \ldots, T_{m} \in \mathbb{R}^{n}$ и матрииа $A$ такие, что степенное преобразование (5.1) приводит систему уравнений

$$
X^{T_{i}} f_{i}(X)=0, \quad i=1, \ldots, m,
$$

$\kappa в и \partial y$

$$
g_{i}(Y)=0, \quad i=1, \ldots, m,
$$

где для всех точек $\widetilde{Q}$ носителей $\mathbf{S}\left(g_{i}\right)$ имеем свойство (5.17).

Tеорема 13. Если у всех точек $\widetilde{Q}$ носителей $\mathbf{S}\left(g_{i}(X)\right), i=1, \ldots, m$, дифференциальных полиномов $g_{1}(Y), \ldots, g_{m}(Y)$ выполнено свойство (5.17), то после логарифмического преобразования (6.1) получаются дифференциальные полиномы $\widetilde{g}_{i}(Z)=g_{i}(Y), i=1, \ldots, m$.

При $n^{\prime}=1$ производная (8.1) является обыкновенной и дифференциальные уравнения системы (8.6) также являются обыкновенньми. Пусть для зависимой переменной $x_{j}, j>1$, максимальный порядок дифференцирования, который имеется в системе (8.6), равен $m_{j}$. Назовем его порядком системы (8.6) по координате $x_{j}$. Величину $m^{\prime \prime}=m_{2}+\cdots+m_{n}$ назовем порядком системы (8.6).

ТЕОРема 14. Пусть $n^{\prime}=1$, система ОДУ (8.6) имеет порядок $m^{\prime \prime}$ и размерность $d<n$. Тогда посредством степенных и логарифмических преобразований эту систему можно свести к системе порядка $m^{\prime \prime}-(n-d)$.

ТЕОРема 15. Пусть $n^{\prime}=1$, система ОДУ (8.6) имеет порядок $m_{j}$ по координате $x_{j}, j=2, \ldots, n$, общий порядок $m^{\prime \prime}=m_{2}+\cdots+m_{n}$ и размерность $d<n$. Пусть $N_{1}, \ldots, N_{n-d}-$ базис нормального подпространства системы (8.6). Пусть решение любой краевой задачи $(8.6), x_{j}^{(k)}(0)=\alpha_{j k}, k=0, \ldots, m_{j}-1 ; j=2, \ldots, n$, существует и единственно. Пусть заданы граничные условия

$$
x_{j}^{(k)}(0)=a_{j k} \neq \infty ; \quad x_{j}^{(l)}(\infty)=b_{j l} \neq 0, \infty,
$$

где для каждого $j$ всего таких условий $m_{j} u 0 \leqslant k, l<m_{j}$, причем из $m^{\prime \prime}$ констант $a_{j k}$ и $b_{j l}$ не менее $n-d$ отличных от нуля, включая все $b_{j l}$. Каждой константе $a_{j k} \neq 0$ ставится в соответствие вектор $R(j, k)=-k E_{1}+E_{j}$, аконстанте $b_{j l}$ - вектор $R(j, l)=-l E_{1}+E_{j}$. Пусть это будут векторы $R_{1}, \ldots, R_{s}$. Образуем матричу

$$
\mathscr{R}=\left(\begin{array}{ccc}
\left\langle N_{1}, R_{1}\right\rangle & \ldots & \left\langle N_{n-d}, R_{1}\right\rangle \\
\ldots \ldots \ldots \ldots \ldots & \ldots \ldots \ldots \ldots \\
\left\langle N_{1}, R_{s}\right\rangle & \ldots & \left\langle N_{n-d}, R_{s}\right\rangle
\end{array}\right) .
$$

Eсли $\operatorname{rank} \mathscr{R}=n-d$, то решение указанной граничной задачи с условиями в нуле $x_{1}=0$ и бесконечности $x_{1}=\infty$ сводится $к$ решению двух граничных задач $c$ условиями только в нуле. 
ПримеР 12. Покажем, что в ситуациях теорем 7 и 8 условия теоремы 15 выполнены. В теореме 7 имеем $n-d=1$ и единственное ненулевое граничное условие есть (7.4). Ему соответствует вектор $R=(-l, 1)$. Скалярное произведение $\langle\Lambda, R\rangle=-l \lambda_{1}+\lambda_{2}$. По условию теоремы 7 оно отлично от нуля.

В теореме 8 имеем $n-d=2$, нормальное подпространство - это вся плоскость $\mathbb{R}_{*}^{2}$, базис в нем образуют векторы $N_{1}=E_{1}$ и $N_{2}=E_{2}$. Два ненулевых граничных условия суть (7.9). Им соответствуют векторы $R_{1}=(2-m, 1), R_{2}=(1-m, 1)$. Матрица $\mathscr{R}$ есть

$$
\left(\begin{array}{ll}
2-m & 1 \\
1-m & 1
\end{array}\right)
$$

Ее определитель равен единице. Следовательно, ее ранг равен двум, т.е. $n-d$.

\section{$\S$ 9. Уровни сложности}

При решении задач с помошью степенной геометрии приходится использовать те или иные объекты в пространстве показателей степеней $\mathbb{R}^{n}$ и в сопряженном пространстве $\mathbb{R}_{*}^{n}$. Дадим классификацию сложности задач, основанную на классификации сложности этих геометрических объектов. Ниже приводится эта последняя классификация, расположенная в порядке возрастания сложности.

І уровень. Линейные подпространства и многообразия, т.е. линейные равенства для векторных степеней.

II уровень. Линейные замены координат.

III уровень. Выпуклые многогранники, их грани и нормальные конусы, т.е. локальные свойства границ многогранников, описываемые линейными неравенствами.

IV уровень. Глобальные свойства вьпуклых многогранников (объем, смешанньй объем и т.п.).

Задачи первых трех уровней сложности обсуждаются ниже в $\S \S 10,11$ и 12 соответственно для систем уравнений следующих типов. Четвертьй уровень обсуждается в замечании 5 в конще $\S 12$.

\section{1. Система алгебраических уравнений}

$$
0=f_{i}(X) \stackrel{\text { def }}{=} \sum f_{i Q} X^{Q} \text { по } Q \in \mathbf{S}_{i}, \quad i=1, \ldots, m .
$$

Здесь каждое уравнение имеет свой носитель $\mathbf{S}_{i}=\mathbf{S}\left(f_{i}\right) \subset \mathbb{R}^{n}$.

\section{2. Система обыкновенных дифференциальных уравнений}

$$
0=f_{i}(X) \stackrel{\text { def }}{=} \sum a_{i Q}(X) \text { по } Q \in \mathbf{S}_{i}, \quad i=1, \ldots, m .
$$

Здесь $x_{1}, \ldots, x_{n_{1}}$ суть параметры, $x_{l}$ - независимая переменная $\left(l=n_{1}+1\right), x_{l+1}, \ldots$, $x_{n}$ - зависимые переменные, $n-l \leqslant m$ и $f_{i}(X)$ суть суммы дифференциальных мономов $a_{i Q}(X)$, каждый из которых является произведением обычного монома $T^{R}$ и производных вида $d^{k} x_{j} / d x_{l}^{k}$ с $j>l$ и имеет свой показатель степени $Q=Q(a)$ [30; гл. VI, $\left.\S 1\right]$. Здесь каждое уравнение имеет свой носитель $\mathbf{S}_{i}=\mathbf{S}\left(f_{i}\right) \subset \mathbb{R}^{n}$. 
3. Система уравнений в частных производных вида $(9.2)$, где $x_{1}, \ldots, x_{n_{1}}$ суть параметры, $x_{n_{1}+1}, \ldots, x_{n_{1}+n_{2}}$ суть независимые переменные, $x_{n_{1}+n_{2}+1}, \ldots, x_{n}$ суть зависимые переменные и $n_{3} \stackrel{\text { def }}{=} n-n_{1}-n_{2} \leqslant m$. При этом $f_{i}(X)$ суть суммы дифоференциальных мономов $a(X)$, каждый из которых является произведением обычного монома $X^{T}$ и производных вида $\partial^{\|K\|} x_{j} / \partial X_{2}^{K}$, где $K=\left(k_{n_{1}+1}, \ldots, k_{n_{1}+n_{2}}\right) \in \mathbb{Z}_{+}^{n_{2}}$, $X_{2}=\left(x_{n_{1}+1}, \ldots, x_{n_{1}+n_{2}}\right), j>n_{1}+n_{2}$, и имеет свой показатель степени $Q(a)$ [25]-[29], [30; гл. VI, § 1], [31]. Здесь каждое уравнение имеет свой носитель $\mathbf{S}_{i}=\mathbf{S}\left(f_{i}\right)$.

В задачах типа 2 (системы ОДУ) выделим два подтипа, которые представляют особый интерес для автора.

2*. Автономная система обыкновенных дифференциальных уравнений, разрешенных относительно производных

$$
d X / d t \stackrel{\text { def }}{=} \dot{X}=\Phi(X) .
$$

Эта система записьвается в виде

$$
(\ln X)=F(X) \stackrel{\text { def }}{=} \sum F_{Q} X^{Q} \text { по } Q \in \mathbf{S}
$$

(см. [10]-[13], [18], [30; гл. III, §2]). Здесь вся система имеет один носитель $\mathbf{S}$. Cреди координат $x_{j}$ могут быть параметры, ибо параметр $x_{j}$ удовлетворяет уравнению $\dot{x}_{j}=0$.

$2^{* *}$. Система Гамильтона $\mathrm{c} l$ степенями свободы

$$
\dot{x}_{i}=\partial h / \partial x_{i+l}, \quad \dot{x}_{i+l}=-\partial h / \partial x_{i}, \quad i=1, \ldots, l,
$$

где $2 l=n$, и функция Гамильтона

$$
h=\sum h_{Q} X^{Q} \text { по } Q \in \mathbf{S}(h) \subset \mathbb{R}^{n} .
$$

Этой системе соответствуют два носителя: носитель $\mathbf{S}(F)$ системы $(9.3)$ типа $2^{*}$ и носитель $\mathbf{S}(h)$ функции Гамильтона $h(X)$.

В задачах типа 3 (системы УрЧП) выделим один подтип, при изучении которого использовался подход степенной геометрии.

\section{$3^{* *}$. Линейное уравнение в частных производных:}

$$
g(D) u=g_{0}\left(x_{1}, \ldots, x_{m}\right)
$$

где $D=\left(\partial / \partial x_{1}, \ldots, \partial / \partial x_{m}\right)$ - векторный дифференциальный оператор, $g(Y)$ - символьньй полином

$$
g(Y)=\sum a_{K} Y^{K} \text { по } K \in \mathbf{S} \subset \mathbb{Z}^{m}
$$

и $g_{0}$ - достаточно хорошая функция. Здесь $n_{1}=0, n_{2}=m, n_{3}=1, X=\left(x_{1}, \ldots, x_{m}, u\right)$. Носитель $\mathbf{S}_{1}$ левой части уравнения (9.7) состоит из точек

$$
\left\{Q=(-K, 1) \text { по } a_{K} \neq 0\right\} .
$$


Поскольку у всех точек $Q$ носителя $\mathbf{S}_{1}$ одинакова последняя координата $q_{m+1}=1$, то ею можно пренебречь и левой части уравнения (9.7) можно сопоставить носитель $-\mathbf{S}$, т.е. носитель символьного многочлена $(9.8)$, взятый со знаком минус.

В дальнейшем предполагаем, что $X \in \mathbb{R}^{n}$ или $\mathbb{C}^{n}$, если все показатели степени целочисленные, и $X \in \mathbb{R}_{+}^{n}$, если они только вещественные.

\section{§10. Линейные равенства}

Пусть в $\mathbb{R}^{n}$ задано множество $\mathbf{S}$ и его аффинная оболочка AFF $\mathbf{S}$ (см. [30; гл. I, $\S 1])$ имеет размерность $d=\operatorname{dim} \mathrm{AFF} \mathbf{S} \stackrel{\text { def }}{=} d(\mathbf{S})$. Тогда в сопряженном пространстве $\mathbb{R}_{*}^{n}$ имеется подпространство $\mathbf{N}(\mathbf{S})$, нормальное к линейному многообразию $\mathrm{AFF} \mathbf{S}$, и $\operatorname{dim} \mathbf{N}(\mathbf{S})=n-d$. Пусть векторы

$$
\Lambda_{i}=\left(\lambda_{i 1}, \ldots, \lambda_{i n}\right), \quad i=1, \ldots, n-d,
$$

образуют базис нормального подпространства $\mathbf{N}(\mathbf{S})$. Тогда многообразие AFF $\mathbf{S}$ определяется системой уравнений

$$
\left\{Q:\left\langle\Lambda_{i}, Q\right\rangle=c_{i}, i=1, \ldots, n-d\right\}
$$

Если в $\mathbb{R}^{n}$ задана совокупность множеств

$$
\mathbf{S}_{1}, \ldots, \mathbf{S}_{m}
$$

то каждое из них имеет свое нормальное подпространство $\mathbf{N}\left(\mathbf{S}_{i}\right)$. Пересечение

$$
\mathbf{N} \stackrel{\text { def }}{=} \mathbf{N}\left(\mathbf{S}_{1}\right) \cap \cdots \cap \mathbf{N}\left(\mathbf{S}_{m}\right)
$$

является нормальным пространством совокупности множеств (10.2). Пусть $e=$ $\operatorname{dim} \mathbf{N}$, тогда его коразмерность $d=n-e$ является размерностью совокупности (10.2). Пусть векторы (10.1) образуют базис нормального подпространства (10.3). Тогда матрица $\left(\Lambda_{1} \ldots \Lambda_{e}\right)^{*}$ имеет ранг $e$. Предположим, что у этой матрицы левый минор порядка $е$ отличен от нуля, т.е.

$$
\operatorname{det}\left(\begin{array}{ccc}
\lambda_{11} & \ldots & \lambda_{1 e} \\
\ldots & \ldots & \ldots \\
\lambda_{e 1} & \ldots & \lambda_{e e}
\end{array}\right) \neq 0
$$

Теперь через единичные точки $E_{e+j}, j=1, \ldots, d$, проведем линейные многообразия $\mathbf{M}_{j}$, нормальные к $\mathbf{N}$. Тогда каждое многообразие $\mathbf{M}_{j}$ определяется системой уравнений

$$
\left\{Q:\left\langle\Lambda_{i}, Q\right\rangle=\lambda_{e+j}, i=1, \ldots, e\right\}
$$

Оно пересекает координатное подпространство $q_{e+1}=\cdots=q_{n}=0$ в точке

$$
R_{j} \stackrel{\text { def }}{=}\left(r_{j 1}, \ldots, r_{j e}, 0, \ldots, 0\right)
$$


являюшейся единственным решением системы уравнений

$$
\lambda_{i 1} r_{j 1}+\cdots+\lambda_{i e} r_{j e}=\lambda_{i e+j}, \quad i=1, \ldots, e .
$$

Разобьем каждый $n$-вектор $X$ на два подвектора

$$
X^{\prime}=\left(x_{1}, \ldots, x_{e}\right) \text { и } X^{\prime \prime}=\left(x_{e+1}, \ldots, x_{n}\right) .
$$

Тогда $R_{j}=\left(R_{j}^{\prime}, 0\right)$.

1. Система алгебраических уравнений. В системе (9.1) каждое уравнение имеет свой носитель $\mathbf{S}_{i}$, и всей системе (9.1) соответствует совокупность носителей (10.2) и нормальное подпространство (10.3). Если его размерность $e>0$, то система (9.1) квазиоднородна. Пусть векторы (10.1) образуют базис в $\mathbf{N}$ и выполнено неравенство (10.4). Тогда система уравнений (9.1) имеет квазиоднородные решения вида

$$
x_{e+j}=b_{j} X^{\prime R_{j}^{\prime}}, \quad j=1, \ldots, d,
$$

где векторы $R_{j}^{\prime}$ суть нетривиальные части векторов $(10.5), b_{j}$ суть ненулевые константы, удовлетворяющие системе уравнений

$$
f_{i}\left(B^{\prime}, B^{\prime \prime}\right)=0, \quad i=1, \ldots, m
$$

и $B^{\prime}=(1, \ldots, 1), B^{\prime \prime}=\left(b_{e+1}, \ldots, b_{n}\right)$. Более того, для алгебраической системы $(9.1)$ квазиоднородные решения (10.6) исчерпьвают все ее решения, лежашие вне координатных гиперплоскостей $x_{i}=0$.

Частные случаи этой ситуации были известны давно, особенно для системы однородных уравнений [91].

2. Система ОДУ. Обозначим $n_{2} \stackrel{\text { def }}{=} 1, n_{3} \stackrel{\text { def }}{=} n-n_{1}-n_{2}$ и предположим, что в системе ОДУ $(9.2) n_{3} \leqslant d<n$, т.е. размерность совокупности носителей (10.2) не менше числа зависимых переменных. Пусть векторы (10.1) образуют базис нормального подпространства (10.3) и выполнено свойство (10.4).

Если $n_{3}=d$, то система (9.2) имеет квазиоднородные решения вида (10.6), где $b_{j}$ суть константы, удовлетворяюшие системе алгебраических уравнений. Примеры таких решений см. в конце примера 5 здесь, в [10], [12] и в 33 гл. VI [30].

Если $n_{3}<d$, то $e \leqslant n_{1}$ и вектор $X^{\prime}$ состоит только из параметров. В этом случае система (9.2) имеет квазиоднородные решения вида (10.6) с $j=l+1, \ldots, n$, где $b_{j}$ суть функции от новой независимой переменной

$$
\xi_{l}=x_{l} / X^{\prime R_{l-e}^{\prime}}
$$

удовлетворяюшие системе ОДУ с $n_{1}-e$ новыми параметрами

$$
\xi_{k}=x_{k} / X^{R_{k-e}^{\prime}}, \quad k=e+1, \ldots, n_{1} .
$$


Эти функции $b_{l+1}\left(\xi_{l}\right), \ldots, b_{n}\left(\xi_{l}\right)$ удовлетворяют системе ОДУ с числом параметров $n_{1}-e<n_{1}$.

Кроме того, для системы ОДУ $(9.2)$ с $d<n$ одну граничную задачу с условиями на двух концах интервала независимой переменной можно заменить двумя граничными задачами на одном конце интервала (см. теоремы $7,8,15)$.

3. Система уравнений в частных производных. Пусть в системе УрЧП (9.2) $d \geqslant n_{3}$, т.е. $e \leqslant n_{1}+n_{2}$. Пусть векторы (10.1) образуют базис нормального подпространства (10.3) системы (9.2) и вьполнено неравенство (10.4).

Если $d=n_{3}$, т.е. $e=n_{1}+n_{2}$, то система (9.2) имеет автомодельные решения вида (10.6), где $b_{j}-$ константы, удовлетворяющие системе алгебраических уравнений.

Если $n_{1} \leqslant e<n_{1}+n_{2}$, то система (9.2) имеет автомодельные решения вида (10.6) с $j=n_{1}+n_{2}+1, \ldots, n$, где $b_{j}$ суть функции от $n_{1}+n_{2}-e$ новых независимых переменных

$$
\xi_{k}=x_{k} / X^{\prime R_{k-e}^{\prime}}, \quad k=e+1, \ldots, n_{1}+n_{2} .
$$

Эти $n_{3}$ функций $b_{j}$ удовлетворяют системе УрЧП с $n_{1}+n_{2}-e$ независимыми переменными и без параметров.

Если $e<n_{1}$, то вектор $X^{\prime}$ состоит из параметров, и система (9.2) имеет автомодельные решения вида (10.6) с $j=n_{1}+n_{2}+1, \ldots, n$, где $b_{j}$ суть функции от $n_{2}$ новых независимых переменных вида $(10.8)$ с $k=n_{1}+1, \ldots, n_{1}+n_{2}$. Эти $n_{3}$ новых функций удовлетворяют системе УрЧП с $n_{1}-e$ новыми параметрами вида (10.7) с $k=e+1, \ldots, n_{1}$. Это следует из теорем 2 и 10 .

Примеры таких автомодельных решений см. в примерах 4, 5, 11. Здесь указано наименьшее возможное количество независимых переменных и параметров автомодельных решений, но можно искать автомодельные решения с большим их числом (см. пример 4).

2* Автономная система ОДУ. Если у системы (9.3) размерность $d<n$, векторы (10.1) образуют базис ее нормального подпространства и вьполнено свойство (10.4), то система (9.3) имеет частные интегралы (т.е. инвариантные многообразия) вида (10.6), где $b_{j}$ суть константы, удовлетворяюшие системе алгебраических уравнений.

К системам вида $(9.3)$ с $d<n$ относится резонансная нормальная форма системы такого же вида в окрестности элементарной неподвижной точки [11], [13], [18]. У нормальной формы (9.3) в записи (9.4) все векторные показатели $Q$ удовлетворяют уравнению

$$
\langle\Lambda, Q\rangle=0
$$

где вектор $\Lambda=\left(\lambda_{1}, \ldots, \lambda_{n}\right)=F_{0}$ образован собственньми значениями матрицы линейной части исходной системы. Для нормальной формы (9.3) всякий ее формальный (или аналитический в нуле) первый интеграл

$$
h=\sum h_{Q} X^{Q} \text { по } Q \in \mathbb{Z}_{+}^{n}
$$

содержит только резонансные мономы $h_{Q} X^{Q}$, удовлетворяющие уравнению (10.9). В частности, если уравнение (10.9) не имеет решений $Q \in \mathbb{Z}_{+}^{n}, Q \neq 0$, то исходная 
система ОДУ, для которой система (9.3) является ее нормальной формой, не имеет формального (и аналитического в неподвижной точке) первого интеграла. Если уравнение (10.9) имеет $d$ линейно независимых решений $Q \in \mathbb{Z}_{+}^{n}$, то исходная система имеет не более $d$ независимых первых интегралов, аналитических в неподвижной точке. Отсюда следует аналитическая неинтегрируемость систем вида (9.3), установленная для различных случаев.

Другой класс систем (9.3) c $d<n$ это системы, инвариантные относительно непрерывной группы линейных преобразований. Например, если система (9.3) инвариантна относительно всех врашений в плоскости $x_{1}, x_{2}$, то в записи (9.4) у всех векторов $Q$ выполнено соотношение $q_{1}=q_{2}$, т.е. носитель системы (9.4) удовлетворяет уравнению (10.9) с $\Lambda=(1,-1,0, \ldots, 0)$. Такие системы встречаются в гидродинамике после редукции эволюционного уравнения на центральное многообразие [1]-[3].

$\mathbf{2}^{* *}$. Система Гамильтона. Если система Гамильтона (9.5) является нормальной формой, то у нее вектор $\Lambda$ имеет вид $\Lambda=\left(\Lambda^{\prime},-\Lambda^{\prime}\right)$, где $\Lambda^{\prime}=\left(\lambda_{1}, \ldots, \lambda_{l}\right)$, и разложение функции Гамильтона (9.6) содержит только резонансные члены, удовлетворяющие уравнению (10.9), т.е.

$$
\left\langle\Lambda^{\prime}, Q^{\prime}-Q^{\prime \prime}\right\rangle=0
$$

где $Q=\left(Q^{\prime}, Q^{\prime \prime}\right)$. Если уравнение $\left\langle\Lambda^{\prime}, Q^{\prime}\right\rangle=0$ имеет ровно $k$ линейно независимых решений $Q_{j}^{\prime} \in \mathbb{Z}^{l}, j=1, \ldots, k$, то система Гамильтона (9.5) имеет $l-k$ независимых первых интегралов вида

$$
\left\langle K^{\prime}, \rho\right\rangle,
$$

где $\left\langle K^{\prime}, Q_{j}^{\prime}\right\rangle=0, j=1, \ldots, k, \varrho_{i}=x_{i} x_{i+l}, \rho=\left(\varrho_{1}, \ldots, \varrho_{l}\right)[22]$.

Оказалось, что равенство (10.10) выполнено для классических гамильтонианов (9.6), соответствующих квантовьм гамильтонианам некоторых задач квантовой оптики [103], [104], [105; §2]. При этом квадратичным интегралам (10.11) соответствуют законы сохранения комбинационного рассеивания. Интегралы (10.11) квадратичны по $X$, но линейны по $\rho$. В $[105 ; \S 5]$ отмечены похожие на $(10.11)$ линейные интегралы дискретных моделей уравнения Больцмана.

\section{§ 11. Линейные преобразования}

В результате степенного преобразования

$$
\ln Y=A \ln X, \quad \operatorname{det} A \neq 0
$$

система уравнений по $X$ некоторого типа переходит в систему уравнений по $Y$ этого же типа (см. [10]-[13], [18], [30; гл. II, $\S \S 3,7$; гл. III, $\S 2 ;$ гл. VI, $\S 2]$ и теоремы 3 и 11 этой статьи). При этом носители уравнений испытьвают аффинное преобразование $\widetilde{Q}=A^{*-1} Q$, а объекты в сопряженном пространстве $\mathbb{R}_{*}^{n}$ испытывают преобразование $\widetilde{P}=A P$. Поэтому с помошью степенного преобразования и сокрашений систему размерности $d$ можно привести к системе, у которой носитель расположен в 
$d$-мерном координатном подпространстве, т.е. у всех векторных показателей степеней $\widetilde{Q}=\left(\widetilde{q}_{1}, \ldots, \widetilde{q}_{n}\right) \in \widetilde{\mathbf{S}}_{i}$ тождественно равны нулю $n-d$ координат $\widetilde{q}_{j}$.

1. Система алгебраических уравнений. Умножая каждое из уравнений системы (9.1) на $X^{T_{i}}$ и делая нужное степенное преобразование (11.1), приведем систему (9.1) к виду

$$
g_{i}\left(y_{1}, \ldots, y_{d}\right)=0, \quad i=1, \ldots, m,
$$

где $g_{i}$ многочлены (см. [7], [34] и [30; гл. II, $\left.\left.\S \S 3,7\right]\right)$.

Частные случаи степенных преобразований алгебраических уравнений использовались давно. Так, для разрешения особенностей использовался сигма-процесс [18], [91], [110]. Это конечная последовательность степенных преобразований (11.1) с матрищами вида $A=E \pm A_{i j}$, где $E$ - единичная матрица и у матрицы $A_{i j}$ элемент на пересечении $i$-й строки и $j$-го столбца равен единице, а остальные элементы равны нулю. Произведение матрищ такого вида является унимодулярной матрицей.

2. Система ОДУ. Для системы ОДУ (9.2) степенные преобразования (11.1) удобно брать лишш с блочнотреугольной матрицей

$$
A=\left(\begin{array}{cc}
A_{11} & 0 \\
A_{12} & A_{22}
\end{array}\right),
$$

где квадратная матрища $A_{11}$ имеет размер $n_{1}$, соответствующий числу параметров, чтобы новые параметры зависели только от старых параметров и не зависели от старых переменных. При этом надо стремиться к тому, чтобы, во-первых, максимально возможное количество новых параметров входило в преобразованную систему в нулевых степенях, т.е. не входило вовсе, и, во-вторых, по возможности у большего количества новых зависимых переменных $y_{j}$ с $j>l$ показатели $\widetilde{q}_{j}$ в носителях всех уравнений равнялись нулю. Тогда эти координаты $y_{j}$ можно заменить на $d \ln y_{j} / d y_{l}$ и понизить порядок системы по этой координате (см. теоремы 6 и 14, пример 10).

Следует отметить, что, вообше говоря, при степенном преобразовании (11.1) дифференциальный полином $f_{i}(X)$ переходит в отношение двух дифференциальных полиномов $f_{i}(X)=g_{i}(Y) / h(Y)$, где носитель $\mathbf{S}(h)$ состоит из одной точки - нуля. Поэтому система уравнений (9.2) переходит в систему $g_{i}(Y)=0, i=1, \ldots, m$. Если в матрище $A_{22}$ в $(11.2)$ верхняя строчка имеет вид $(a, 0, \ldots, 0)$, т.е. новая независимая переменная не зависит от старых зависимых переменных, то $h(Y) \equiv 1$ (см. [30; гл. VI, $\S 2]$ и здесь $\S 5$, замечание 1$)$.

3. Система УрЧП. Для нее степенное преобразование (11.1) надо брать с блочнотреугольной матрищей (11.2). Кроме того, при степенном преобразовании надо стремиться к тому, чтобы у носителей преобразованных уравнений было больше нулевых координат, соответствующих параметрам и независимым переменным. В преобразованной системе это уменьшает количество параметров и сушественных независимых переменных. Ибо для независимой переменной $y_{j}$ с нулевым показателем в носителях можно сделать логарифмическое преобразование $z_{j}=\ln y_{j}$, не нарушая характера уравнений как дифференциальных многочленов (см. [26]-[29], [30; гл. VI, §3], [31] и здесь $\S 6)$. Решения, которые не зависят от этих независимых переменных $z_{j}$, являются автомодельными. 
$2^{*}$. Автономная система ОДУ. С помошњю степенного преобразования (11.1) и замены времени вида $d \tilde{t}=X^{T} d t$ систему (9.4) можно привести к виду

$$
\left(\ln y_{i}\right)=g_{i}\left(y_{1}, \ldots, y_{d}\right), \quad i=1, \ldots, n \text {. }
$$

У этой системы отшепляется подсистема для $y_{1}, \ldots, y_{d}$. Остальные $y_{j}$ получаются из них с помошью квадратур (см. [10]-[13], [18], [30; гл. III, § 2]).

В частности, для нормальной формы $(9.4),(10.9)$ размерность $d$ не превосходит кратности резонанса. Поэтому с помошью степенного преобразования ее интегрирование сводится к решению системы порядка $d$ (см. [11], [13], [18]).

$2^{* *}$. Система Гамильтона. Вообще говоря, степенное преобразование (11.1) не является каноническим и разрушает гамильтонову структуру системы (9.5). В [22; гл. I, §3] указано каноническое преобразование, которое понижает число степеней свободы системы Гамильтона (9.5), если $\operatorname{dim} \operatorname{AFF} \mathbf{S}(h)<n$. А именно, если уравнение $\left\langle\Lambda^{\prime}, Q^{\prime}\right\rangle=0$ имеет $k$ линейно независимых решений $Q^{\prime} \in \mathbb{Z}^{l}$, то система Гамильтона (9.5) приводится к системе Гамильтона с $k$ степенями свободы и $l-k$ параметрами.

ЗАмечание 3 . Алгоритмы вычисления матрицы $A$ для степенного преобразования (11.1), приводящего систему к указанным упрошениям, описаны в [30; гл. I, §11].

\section{$\S$ 12. Линейные неравенства}

Теперь рассматриваются алгебраические и дифференциальные уравнения, носители которых не обязаны лежать в линейном многообразии меньшей размерности. Но по носителю $\mathbf{S}(f)$ уравнения $0=f(X)=\sum a_{Q}(X)=0$ строится его вьпуклая оболочка $\boldsymbol{\Gamma}$. Поверхность $\partial \Gamma$ многогранника $\boldsymbol{\Gamma}$ состоит изграней $\boldsymbol{\Gamma}_{j}^{(d)}$ разных размерностей $d$. Каждой грани $\boldsymbol{\Gamma}_{j}^{(d)}$ соответствует граничное подмножество $\mathbf{S}_{j}^{(d)}=\Gamma_{j}^{(d)} \cap \mathbf{S} \subset \mathbb{R}^{n}$ и нормальньй конус $\mathbf{U}_{j}^{(d)} \subset \mathbb{R}_{*}^{n}$. Кроме того, каждой грани $\boldsymbol{\Gamma}_{j}^{(d)}$ соответствует укороченное уравнение

$$
0=\widehat{f}_{j}^{(d)}(X) \stackrel{\text { def }}{=} \sum a_{Q}(X) \text { по } Q \in \mathbf{S}_{j}^{(d)}
$$

и множество $\mathscr{U}_{j}^{(d)}(\varepsilon)$ в $X$-пространстве. При этом укороченное уравнение $(12.1)$ является первым приближением уравнения $f(X)=0$ в множестве $\mathscr{U}_{j}^{(d)}(\varepsilon)$ (см. [18]).

Для полной системы уравнений таким образом выделяется укороченная система, которая имеет размерность $d<n$, свой нормальный конус и свое множество типа $\mathscr{U}(\varepsilon)$, где она является первым приближением полной системы. При этом если полная система имеет решение, лежашее в множестве $\mathscr{U}(\varepsilon)$, то его первое приближение является решением соответствуюшей укороченной системы [25], [27]-[31]. Это позволяет последовательно находить асимптотики решений и их асимптотические разложения. Для нахождения решений укороченных систем можно использовать степенные и логарифмические преобразования, как описано вьше. Именно этим вопросам для разных типов уравнений посвящены гл. II-VI [30] (см. также [27]-[29], [31]). 
А именно, системы алгебраических уравнений (9.1) рассмотрены в гл. II. Системы обыкновенных дифференциальных уравнений - в $\S 1-4$ гл. VI. Общие системы уравнений в частных производных - в $\S ~ 1,5,6$ гл. VI. Системы уравнений вида (9.3) - в гл. III и V , а системы Гамильтона (9.5) - в гл. IV.

B [4] и [30; гл. I] описаны алгоритмы вычисления граничных подмножеств (т.е. укороченных систем) и их нормальных конусов.

Перечислим применения степенной геометрии, не вошедшие в книгу [30].

2*. Автономная система ОДУ. Для систем ОДУ (9.3) типа 2* эта техника позволяет вычислять асимптотические разложения не только для асимптотических решений, но и для нейтральных (периодических, условно периодических и т. д.) [18]. Для этого по укороченной системе находится степенное преобразование, приводящее ее к системе вида (11.3). Это степенное преобразование делается в полной системе. Затем в окрестности каждой элементарной особой точки с $y_{d+1}=\cdots=y_{n}=0$ полная система приводится к нормальной форме. Если нужно, в нормальной форме делается другое степенное преобразование. В [21], [86] этот подход впервые был использован для анализа решений конкретных систем. В [39], [40], [42], [43] и в гл. V [30] этим методом изучена конкретная система вида (9.3). В [3] этот же подход использован для другой системы (9.3), возникшей в гидродинамической задаче.

Кроме того, в [13] при доказательстве расходимости нормализующего преобразования для систем типа (9.3) использовались соображения степенной геометрии для выделения первых приближений этих преобразований и явных оценок тейлоровских коэффициентов выделенных первых приближений. В [24] предпринята попытка популярно объяснить эти геометрические конструкции, но они до сих пор остались не понятыми. Для двумерных вешественных систем (9.3) в [19], [20] даны доказательства расходимости нормализующего преобразования, также используюшие степенную геометрию.

Для линейной неавтономной системы ОДУ вида $\dot{X}=\Phi(t) X$ степенная геометрия также позволяет получить новые результаты и интерпретировать старые (см. [13; введение], [19], [20], [32]).

ЗАмЕЧАнИЕ 4. В [10], [12], [18] и в гл. III [30] процедура вычисления асимптотик решений системы (9.3) основана на рассмотрении в $\mathbb{R}^{n}$ одного носителя всей системы. Возможно, более универсальным является обший подход, согласно которому каждому уравнению системы (9.3) ставится в соответствие свой носитель в $\mathbb{R}^{n+1}$ и укороченные системы находятся по этим носителям согласно общим правилам.

$\mathbf{3}^{*}$. Линейное уравнение в частных производных (9.7). В терминах носителя символьного полинома (9.8) формулировались условия, обеспечивающие локальную разрешимость некоторых задач для уравнения (9.7). Поскольку локальньм свойствам (в окрестности точки $X_{2}^{0}$ ) соответствует конус задачи $\left\{P_{2}<0\right\}$ для носителя $\mathbf{S}_{1}$ дифференциального полинома $g(D) u$ и справедливо равенство $\mathbf{S}(g)=-\mathbf{S}_{1}$, то для носителя $\mathbf{S}(g)$ этим локальным свойствам соответствует конус задачи $\left\{P_{2}>0\right\}$. В работах [9], [53], [55]-[57], [78]-[81], [107]-[109] символьный полином записывается в виде

$$
g(i Z)=a(Z)+i b(Z)=\sum a_{K} Z^{K}+\sum b_{L} Z^{L} .
$$

Очевидно, $\mathbf{S}(g)=\mathbf{S}(a) \cup \mathbf{S}(b)$. Рассматриваются многогранники $\boldsymbol{\Gamma}$ - выпуклая оболочка носителя $\mathbf{S}(a)$ - и $\boldsymbol{\Gamma}_{1}$ - вьпуклая оболочка множества $\mathbf{S}(a) \cup\{0\}$. У многогранника $\boldsymbol{\Gamma}$ 
выделяется его ведушая часть относительно отрицательного ортанта $\mathbb{R}_{-}^{m}=\{K \leqslant 0\}$ : $\widetilde{\boldsymbol{\Gamma}}=\boldsymbol{\Gamma} / \mathbb{R}_{-}^{m}($ см. $[30 ;$ гл. I, $\S 8])$, и у многочлена $a(Z)$ выделяется его главная часть

$$
\widetilde{a}(Z)=\sum a_{k} Z^{k} \text { по } K \in \widetilde{\boldsymbol{\Gamma}} \text {. }
$$

Предполагается, что

(a) множество $\widetilde{\boldsymbol{\Gamma}}$ имеет точки на каждой координатной оси;

(б) каждый укороченный многочлен $\widehat{a}_{j}^{(m-1)}(Z)$, соответствующий гиперграни $\Gamma_{j}^{(m-1)} \subset \widetilde{\boldsymbol{\Gamma}}$ (т.е. с нормальным вектором $P>0$ ), не имеет вещественных корней вне координатных подпространств.

Тогда при вешественном $Z \rightarrow \infty$ вьполняется оценка

$$
|a(Z)| \geqslant c \sum Z^{k} \text { по } K \in \mathbf{V}
$$

где постоянная $c>0$, а $\mathbf{V}$ это множество вершин гиперповерхности $\widetilde{\boldsymbol{\Gamma}}$. Такая оценка позволяет доказать гипоэллиптичность уравнения (9.7) и локальную разрешимость тех или иных задач для дифференциального уравнения (9.7) (см. [9], [53], [55]-[57], [78]-[81], [107]-[109]). В этих работах рассматривалось также такое уравнение (9.7), в котором оператор $g(D)$ зависит от координат $x_{1}, \ldots, x_{m}$. Но эта зависимость предполагалась слабой и не влияюшей на структуру многогранника $\widetilde{\boldsymbol{\Gamma}}$. Кроме того, в [108] рассматривалась система линейных уравнений.

Перечислим некоторые задачи разных дисциплин, в которых были получены новые результаты с помощью степенной геометрии.

1. Алгебраические уравнения. В [37] и [30; гл. II, §9] были изучены особые положения механизмов (робототехника).

2. ОДУ. Изучены семейства периодических решений уравнения плоских колебаний спутника на эллиптической орбите (уравнения Белецкого) и их особенности [44]-[47], [87]-[89] (небесная механика).

3. УрЧП. Дано строгое математическое обоснование теории пограничного слоя в простейшей задаче обтекания (см. [30; гл. VI, §6] и [48]-[50] (гидродинамика). Более сложные задачи с пограничным слоем рассмотрены в [100], [101].

$\mathbf{2}^{* *}$. Система Гамильтона. Особенности пространственных колебаний спутника на круговой орбите [30; гл. IV,$\S 3]$. Особенности семейств периодических решений ограниченной задачи трех тел [17], [30; гл. IV , § 4], [31] (небесная механика).

ЗАмечАниЕ 5. Результаты, используюшие глобальные свойства многогранников, имеются только для системы алгебраических уравнений. Например, такой. В случае обшего положения число решений системы $n$ алгебраических уравнений от $n$ неизвестных равно смешанному объему Минковского многогранников Ньютона этих уравнений [7]. В работах Хованского и его коллег установлены интересные связи между топологической структурой алгебраического многообразия и многогранниками Ньютона уравнений, задаюших эти многообразия [52], [54], [61]-[69], [85]. Основным достижением здесь является открытие связи многогранников Ньютона и торических многообразий, т.е. построение торической компактификации и разрешения особенностей 
для достаточно обших систем алгебраических уравнений с фиксированными многогранниками Ньютона. Эта конструкция позволила применить теорию торических многообразий к многогранникам и привела к решительному прогрессу в этой области. См. обзор работ Хованского [69].

\section{$\S$ 13. Исторические замечания}

Работы, используюшие подходы степенной геометрии, разделяются на 3 струи со своими источниками:

1. Работы автора и его последователей, начиная с [10];

2. Работы В. П. Михайлова и его последователей, начиная с [78];

3. Работы В. И. Арнольда и его последователей, начиная с [5].

Ниже с позиций автора излагается история “течений” в этих струях и их взаимных влияний.

1. В [10] для системы (9.3) введены многогранник системы, его грани, укороченные системы, нормальные конусы, степенные преобразования и применены для вычисления асимптотик асимптотических решений (см. также [12], [14], [15], [18], [30; гл. III]). В [15] этот подход распространен на вычисление ветвей алгебраической кривой вблизи вырожденной критической точки (см. также [16], [18], [30; гл. II], [33]-[36], [93]). В $[15]$ каждому нормальному конусу в $\mathbb{R}_{*}^{n}$ поставлено в соответствие свое множество $\mathscr{U}(\varepsilon)$ в $X$-пространстве и введены степенные ряды, носители которых лежат в заданном конусе (см. также [18]). В [17] с помощью многогранника Ньютона функции Гамильтона были выделены первые приближения для системы Гамильтона вблизи особенности (см. также [23], [30; гл. IV], [31], [41], [95]). В [92] была начата разработка алгоритма для выгисления многогранника, его граней и нормальных конусов по заданному носителю (см. также [94], [4], [30; гл. I]). В [38] подход степенной геометрии был распространен на разрешение особенностей любых алгебраических многообразий (см. также [30; гл. II]). В [25] показано, как с помощью степенной геометрии выделять первые приближения любых систем дифференциальных уравнений и находить асимптотики их решений. В частности, показано, что подходы работ [55]-[57], [78]-[81], [107]-[109] для уравнения (9.7) и результаты работы [102] по сингулярным возмушениям укладьваются в обшую схему (см. также [27]-[29], [30; гл. VI, § 1], [31]). В [26] замечено, что при степенном преобразовании носитель дифференциального полинома испытывает линейное преобразование (см. также [27]-[31] и здесь теорему 3 в $§ 5)$. В [28] введено логарифомическое преобразование.

2. Для дифференциального уравнения (9.7) Михайлов [78] предложил рассматривать носитель его характеристического полинома и многогранник, являющийся выпуклой оболочкой этого носителя и точки нуль (см. конец 12 и также [9], [53], [55]-[57], [79]-[81], [107]-[109]). При этом в начальных работах [78], [79] многогранник не имел названия, в [80], [81] и [108] он назьвался "характеристическим", а начиная с работы [55] и далее - "многогранником Ньютона", хотя обычно ломаную Ньютона применяли в окрестности нуля, и ее вьпуклость, соответствующая главной части полинома, была обращена к нулю. А в задачах с гипоэллиптическими характеристическими полиномами вьпуклость многогранника Ньютона, соответствующая главной части полинома, обращена к бесконечности. 
3. В [5; п. 9.9] Арнольд заметил, что в случае общего положения некоторые локальные свойства функции двух переменных в окрестности вырожденной критической точки зависят только от размеров области, ограниченной осями и выпуклостью ломаной Ньютона этой функции. Это послужило толчком к написанию работ [75], [76], где уже рассматривались многогранники Ньютона одного и нескольких рядов. Интересно отметить, что в этих работах многогранник Ньютона - это не просто выпуклая оболочка носителя, а выпуклая оболочка носителя, сложенного с неотрицательным ортантом $\{Q \geqslant 0\}$, аналогично тому, как в работах [55], [56] многогранник Ньютона это выпуклая оболочка носителя, сложенного с отрицательньм ортантом $\{Q \leqslant 0\}$. Впрочем, аналогичные определения многогранника Ньютона содержатся и в некоторых последующих работах (см., например, [6; п. 6.2]). Явное определение многогранника Ньютона как вьпуклой оболочки носителя имеется в [8], а неявное - в [7]. В дальнейшем этот подход использовался преимушественно для вьгисления асимптотик осциллируюших интегралов [97]-[99], [6] и для изучения свойств решений системы алгебраических уравнений [52], [54], [61]-[69], [74], [77], [85].

Теперь остановимся на взаимном влиянии указанных трех струй. Насколько известно автору, каждая из струй 2 и 3 не оказала заметного идейного влияния на другие струи, только терминологическое. Так, термин многогранник Ньютона, возникший в работе [55] второй струи, стал общепризнанным. Аналогично, стал общепризнанным термин носитель из третьей струи. Поэтому рассмотрим влияние первой струи на вторую и третью.

Влияние первой струи на вторую начинается, повидимому, в 70-е годы. Особенно это заметно в статье [109] и книге [57], где используются введенные в [10], [15], [18] нормальные конусы и множества $\mathscr{U}(\varepsilon)$ (без конкретных ссылок). По-видимому, впервые обе эти струи встретились в [30; гл. IV,$\S 2$, п. 2.4]. В [25; пример 4] и [30; гл. VI, пример 1.6] показано, как степенная геометрия второй струи укладьвается в общую схему.

Влияние первой струи на третью происходило следуюшим образом. В 1960 и 1961 гг. на конкурс студенческих работ мех-мата МГУ были представлены работы автора, послужившие основой для публикаций [10], [11], [14]. Отзывы на эти работы писал В.И. Арнольд, бывший тогда аспирантом. В 1968 г. он был официальным оппонентом по докторской диссертации автора, опубликованной впоследствии в виде статей [13]. У автора сохранился отзьв В.И. Арнольда на рукописи этих статей, написанньй в декабре 1969 г. В нем В.И. Арнольд критикует “геометрию показателей степеней" в трех следующих замечаниях.

9. На стр. 14 автор пишет о “новых понятиях геометрии показателей степеней”. На стр. 18 выясняется, что это - “конусы, многогранники и другие геометрические объекты, которьм нет аналогов в алгебрах Ли”.

Должен признаться, что я в “геометрии показателей” новых понятий не вижу: по моему, следовало написать "новых по мнению автора".

11. На стр. 18 автор пишет: "Мы считаем, что концепции геометрии показателей степеней более плодотворны" (чем точка зрения алгебр Ли). Рецензенту кажется, что высказанное здесь автором мнение объясняется просто его незнакомством с алгебрами Ли и с теми упрошениями, которые обшая групповая точка зрения могла бы внести в его изложение. Цитированную критику алгебр Ли лучше опустить. 
16. На стр. 195-198 автор в следующих терминах говорит о своих работах:

“Доказательства ... основаны исключительно на методах, развитых автором. Суть их в геометрии показателей степеней ...". "Степенные преобразования, введенные автором в 1962 г., делают более содержательной геометрию показателей степеней”.

По мнению рецензента:

А. "Геометрия показателей" состоит в том, что показатели степеней в кратных рядах Фурье (Лорана, Тейлора) изображаются точками целочисленной решетки. Эта геометрия становится "более содержательной", когда используется теорема о приведении целочисленной матрицы к стандартному виду с помощью элементарных преобразований. То и другое общеизвестно и обычно используется безо всякой торжественности.

Б. “Степенные преобразования, введенные автором" широко известны под именем “кратных сигма- и анти сигма-процессов" или "разрешений особенностей" в алгебраической геометрии. В дифференциальных уравнениях ими также давно пользуются (см., например, мемуар Бендиксона 1901 г.).

Рецензенту кажется, что содержашиеся на стр. 195-198 самовосхваления автора следует опустить: читатели (и автор) от этого только вьпграют.

В тексте статей [13] критиковавшиеся места остались в следующем виде. К замечанию 9 относится абзац перед формулировкой условия $\omega$ в п. II введения (с. 127). Текст, указанньй в замечании 11 был выброшен. Замечание 16 относится к первому абзацу п. III, $\S 6$, гл. III (с. 209) и к абзацу перед формулировкой теоремы 10 в том же пункте (с. 210). Таким образом, В. И. Арнольду потребовалось 14 лет (с 1960 г. по 1974 г., см. [5]), чтобы осознать полезность степенной геометрии. Дальнейшеевлияние первой струи на третью происходило в 1973-1976 гг. Так, в работе [7] используются степенное преобразование и связанное с ним линейное преобразование в пространстве показателей степеней (разумеется, без ссылок). Степенные преобразования используются также в $[6 ; \S 8$, п. 8.1. Б] под именем мономиальные отображения и тоже без ссылок. Там же используются аналоги нормальных конусов и другие инструменты степенной геометрии.

\section{СПИСОК ЛИТЕРАТУРЫ}

[1] Афендиков А. Л., Бабенко К. И. Бифуркация рождения цикла в некоторых задачах с симметрией // Докл. АН СССР. 1988. Т. 300. № 1. С. 14-18.

[2] Afendikov A., Mielke A. Bifurcation of homoclinic orbits to a saddle-focus in reversible systems with SO(2)-symmetry // J. Differential Equations. 1999. V. 159. № 2. P. 370-402.

[3] Афрендиков А. Л., Мильке А. О семействе обратимых $\mathrm{SO}(2)$-инвариантных векторных полей с четырехкратным неполупростым нулевым собственным значением // Докл. АH. 1999. Т. 369. №2. C. 154-157.

[4] Арансон А.Б. Вычисление многогранника Ньютона // Материалы Международной конференции и Чебышёвских чтений, посвященных 175 -летию со дня рождения П. Л. Чебышёва. Т. 1. М.: МГУ, 1996. С. 32-34.

[5] Арнольд В. И. Нормальные формы функций в окрестности вырожденных критических точек // УМН. 1974. Т. 29. №2. С. 11-49.

[6] Арнольд В.И., Варченко А.Н., Гусейн-Заде С. М. Особенности дифференцируемых отображений. Т. 2. Монодромия и асимптотики интегралов. М.: Наука, 1984. 
[7] Бернштейн Д. Н. Число корней системы уравнений // Функц. анализ и его прил. 1975. Т. 9. № 3. С. 1-4.

[8] Бернштейн Д. Н., Кушниренко А. Г., Хованский А. Г. Многогранники Ньютона // УМH. 1976. Т. 31. №3. С. 201-202.

[9] Boggiatto P., Buzano E., Rodino L. Global Hypoellipticity and Spectral Theory. Berlin: Akademie Verlag, 1996.

[10] Брюно А. Д. Асимптотика решений нелинейных систем дифференциальных уравнений // Докл. АН СССР. 1962. Т. 143. № 4. С. 763-766.

[11] Брюно А. Д. Нормальная форма дифференциальных уравнений // Докл. АН СССР. 1964. T. 157. №6. C. 1276-1279.

[12] Брюно А. Д. Степенные асимптотики решений нелинейных систем // Изв. АН СССР. Сер. матем. 1965. Т. 29. № 2. С. 329-364.

[13] Брюно А. Д. Аналитическая форма дифференциальных уравнений // Труды ММО. 1971. T. 25. С. $119-262 ;$ 1972. Т. 26. С. 199-238.

[14] Брюно А. Д. О степенных асимптотиках решений нелинейных систем // Препринт № 54. М.: ИПМ АН СССР, 1973.

[15] Брюно А. Д. Элементы нелинейного анализа: Конспект лекций. Самарканд: СамГУ, 1973.

[16] Брюно А. Д. Локальные методы в нелинейном анализе // Функциональный анализ и некоторые вопросы КТДУ / ред. Е. В. Воскресенский. Саранск: Мордовский ун-т, 1976. C. $77-80$.

[17] Брюно А. Д. О периодических облетах Луны // Препринт № 91. М.: ИПМ АН СССР, 1978; Bruno A. D. On periodic flybys of the moon. V. 24. № 3, 1981. P. 255-268.

[18] Брюно А. Д. Локальный методнелинейного анализа дифференциальных уравнений. М.: Наука, 1979.

[19] Брюно А. Д. Расходимость вещественного нормализующего преобразования // Препринт № 62. М.: ИПМ АН СССР, 1979; Bruno A. D. Divergence of the real normalizing transformation. V. 12. № 1, 1993. P. 13-23.

[20] Брюно А. Д. О расходимости вещественного нормализующего преобразования // Матем. заметки. 1982. Т. 31. № 3. С. 403-410.

[21] Брюно А. Д.ББифркация периодических решений в симметричном случае кратной пары мнимых собственных значений // Численное решение обыкновенных дифференциальных уравнений / ред. С. С. Филиппов. М.: ИПМ АН СССР, 1988. С. 161-176.

[22] Брюно А. Д. Ограниченная задача трех тел. М.: Наука, 1990.

[23] Bruno A.D. A local analysis of Hamiltonian systems // Preprint M/1990/33. Paris: I.H.E.S., 1990.

[24] Bruno A.D. On divergence of normalizing transformation // Preprint M 72. Paris: I.H.E.S., 1990.

[25] Брюно А. Д. Первые приближения дифференциальных уравнений // Докл. АН. 1994. T. 335. №4. C. 413-416.

[26] Брюно А. Д. Алгоритмы нелинейного анализа // УМН. 1996. Т. 51. № 5. С. 186.

[27] Bruno A. D. Algorithms of the local nonlinear analysis // Nonlinear Anal. 1997. V. 30. № 7. P. 4683-4694.

[28] Bruno A.D. Power geometry // J. Dynamical Control Systems. 1997. V. 3. № 4 . P. 471-492.

[29] Bruno A. D. Newton polyhedra and power transformations // Math. Comput. Simulation. 1998. V. 45. № 5-6. P. 429-444.

[30] Брюно А. Д. Степенная геометрия в алгебраических и дифференциальных уравнениях. М.: Физматлит, 1998.

[31] Bruno A.D. Power geometry and four applications // J. Math. Sci. 1999. V. 95. № 5. P. 2483-2512.

[32] Брюно А.Д. Мероморфная приводимость линейной треугольной системы ОДУ // Докл. АН. 2000 (в печати).

[33] Bruno A.D., Soleev A. The local uniformization of branches of an algebraic curve // Preprint M 34. Paris: I.H.E.S., 1990. 
[34] Брюно А. Д., Солеев А.Локальная униформизация ветвей пространственной кривой и многогранники Ньютона // Алгебра и анализ. 1991. Т. 3. № 1. С. 67-102.

[35] Bruno A. D., Soleev A. The local uniformization of branches of a space curve // Proc. of the 22 Annual Iranian Math. Conference / ed. M. R. R. Moghaddam and M. A. Pourabdollah. Mashhad: Ferdowsi University, 1991. P. 72-85.

[36] Bruno A. D., Soleev A. The local uniformization of branches of an algebraic curve // Contemp. Math. 1992. V. 131. № 3. P. 361-378.

[37] Брюно А. Д., Солеев А. Классификация особенностей функции положения механизмов // Проблемы машиностроения и надежности машин. 1994. № 1. С. 102-109.

[38] Брюно А. Д., Солеев А. Первые приближения алгебраических уравнений // Докл. АН. 1994. Т. 335. № 3. С. 277-278.

[39] Брюно А. Д., Солеев А. Локальный анализ особенности одной обратимой системы ОДУ // УМН. 1995. Т. 50. №6. С. 169-170.

[40] Брюно А. Д., Солеев А. Бифуркации решений одной обратимой системы ОДУ // Докл. АН. 1995. Т. 345. № 5. С. 590-592.

[41] Брюно А.Д., Солеев А. Гамильтоновы укорочения системы Гамильтона // Докл. PAH. 1996. Т. 349. № 2. C. 153-155.

[42] Bruno A. D., Soleev A. Local analysis of a reversible ODE system and the Newton polyhedron // Nonlinear Anal. 1997. V. 30. № 8. P. 4833-4838.

[43] Брюно А. Д., Солеев А. Локальный анализ особенностей одной обратимой системы ОДУ // Труды ММО. 1998. Т. 59. С. 3-72.

[44] Брюно А. Д., Варин В.П. Фрактальная структура периодических колебаний спутника // Материалы Международной конференции и Чебышёвских чтений, посвященных 175-летию со дня рождения П. Л. Чебышёва. Т. 1. М.: МГУ, 1996. С. 75-77.

[45] Bruno A. D., Varin V.P. The limit problems for the equation of oscillation of a satellite // Celestial Mech. Dynam. Astronom. 1997. V. 67. № 1. P. 1-40.

[46] Bruno A.D., Varin V.P. Singularities of oscillations of a satellite on highly eccentric elliptic orbits // Nonlinear Anal. 1997. V. 30. № 4. P. 2541-2546.

[47] Bruno A.D., Varin V.P. Generalized periodic solutions to the equation of oscillations of a satellite // Z. Angew. Math. Mech. 1999. V. 79. № 2. P. 283-284.

[48] Брюно А. Д., Васильев М.М.Асимптотический анализ обтекания полубесконечной пластины методом многогранника Ньютона // Материалы Международной конференции и Чебышёвских чтений, посвященных 175 -летию со дня рождения П. Л. Чебышёва. Т. 1. М.: МГУ, 1996. С. 78-80.

[49] Bruno A. D., Vasil'ev M. M. Asymptotic analysis of the viscous fluid flow around a flat plate by the Newton polyhedra // Nonlinear Anal. 1997. V. 30. № 8. P. 4765-4770.

[50] Bruno A. D., Vasil'ev M. M. Newton polyhedron and Prandtl equation for a boundary layer // Z. Angew. Math. Mech. 1998. V. 78. № 1. P. 309-310.

[51] Черников С. Н. Линейные неравенства. М.: Наука, 1968.

[52] Данилов В.И., Хованский А. Г. Многогранники Ньютона и алгоритм вычисления чисел Ходжа-Делиня // Изв. АН СССР. Сер. матем. 1986. Т. 50. № 5. С. 925-945.

[53] Friberg J. Multiquasielliptic polynomials // Ann. Scuola Norm. Sup. Pisa. Cl. Sci. (4). 1967. V. 21. № 2. P. 233-260

[54] Гельфонд А. О., Хованский А. Г. Многогранники Ньютона и вычеты Гротендика // Докл. АН. 1996. Т. 350. №3. С. 298-300.

[55] Гиндикин С. Г. Об одном классе дифференциальных операторов, допускающих двустороннюю энергетическую оценку // УМН. 1973. Т. 28. № 6. С. 199-200.

[56] Гиндикин С. Г. Энергетические оценки, связанные с многогранником Ньютона // Труды MMO. 1974. Т. 31. C. 189-236.

[57] Gindikin S., Volevich L. R. The Method of Newton's Polyhedron in the Theory of Partial Differential Equations. Dordrecht: Kluwer Acad. Publ., 1992.

[58] Гурса Э. Курс математического анализа. Т. 1. Ч. 2. М.-Л.: ГТТИ, 1933.

[59] Ибрагимов Н. Х. Группы преобразований в математической физике. М.: Наука, 1983.

[60] Ibragimov N. H. (Ed.) Lie Group Analysis of Differential Equations. V. 1. Boca Raton, FL: CRC Press, 1994. 
[61] Казарновский Б.Я. Многогранники Ньютона и корни систем экспоненциальных сумм // Функц. анализ и его прил. 1984. Т. 18. № 4. С. 40-49.

[62] Хованский А. Г. Многогранники Ньютона и родполных пересечений // Функц. анализ и его прил. 1978. Т. 12. № 1. С. 51-61.

[63] Хованский А.Г. Многогранники Ньютона и формула Эйлера-Якоби // УМН. 1978. T. 33. №6. C. 245-246.

[64] Хованский А.Г. Многогранники Ньютона // Итоги науки и техники. Современные проблемы математики. Т. 22. М.: ВИНИТИ, 1983. С. 206-239.

[65] Хованский А. Г. Многогранник Ньютона, полином Гильберта и суммы конечных множеств // Функц. анализ и его прил. 1992. Т. 29. № 4. С. 57-63.

[66] Хованский А. Г. Многоугольники Ньютона, кривые на торических поверхностях и обращение теоремы Вейля // УМН. 1997. Т. 52. №6. С. 318.

[67] Khovanskii A. G. Consistent partition of polytopes and polynomial measures // Amer. Math. Soc. Transl. Ser. 2. 1997. V. 180. P. 157-166.

[68] Khovanskii A. G. Newton polyhedrons, a new formula for mixed volume, product of roots of a system of equations // Proc. of the Arnoldfest, Fields Institute of Communications, 2000.

[69] Хованский А.Г. Геометрия многогранников и алгебраическая геометрия // http:// www.math.toronto.edu/askold/, 1999.

[70] Klamkin M.S. On the transformation of a class of boundary value problems into initial value problems for ordinary differential equations // SIAM Rev. 1962. V. 4. P. 43-57.

[71] Klamkin M.S. Transformation of boundary value into initial value problems // J. Math. Anal. Appl. 1970. V. 32. № 2. P. 308-330.

[72] Клоков Ю.А., Михайлов А.П., Адъютов М. М. Нелинейные математические модели и неклассические краевые задачи для обыкновенных дифференциальных уравнений // Фундаментальные основы математического моделирования. М.: Наука, 1997. С. 98-197.

[73] Костин В. М. О некоторых инвариантных решениях уравнения типа Кортевега-де Вриза // Журн. прикл. мех. и техн. физ. 1969. № 4. С. 69-73.

[74] Kouchnirenko A. G. Polyedres de Newton et nombres de Milnor // Invent. Math. 1976. V. 32. № 1. P. 1-31.

[75] Кушниренко А. Г. Многогранники Ньютона и число решений системы $k$ уравнений с $k$ неизвестными // УМН. 1975. Т. 30. №2. С. 266-267.

[76] Кушниренко А. Г. Многогранники Ньютона и числа Милнора // Функц. анализ и его прил. 1975. Т. 9. №1. С. 74-75.

[77] Кушниренко А.Г. Многогранники Ньютона и теорема Безу // Функц. анализ и его прил. 1976. Т. 10. № 3. С. $82-83$.

[78] Михайлов В. П. О первой краевой задаче для некоторых полуограниченных операторов // Докл. АН СССР. 1963. Т. 151. № 2. С. 282-285.

[79] Михайлов В.П. О поведении на бесконечности некоторых классов многочленов // Докл. АН СССР. 1965. Т. 164. № 3. С. 499-502.

[80] Михайлов В.П. О поведении на бесконечности одного класса многочленов // Труды МИАН. 1967. Т. 91. С. 59-80.

[81] Михайлов В. П. Первая краевая задача для квазиэллиптических и квазипараболических уравнений // Труды МИАН. 1967. Т. 91. С. 81-99.

[82] Na T. Y. Computational Methods in Engeneering Boundary Value Problems. New York: Academic Press, 1979.

[83] Овсянников Л.В. Групповые свойства уравнения Чаплыгина // Журн. прикл. мех. техн. физ. 1960. № 3. С. 126-135.

[84] Овсянников Л.В.Групповой анализ дифференциальных уравнений. М.: Наука, 1978.

[85] Пухликов А.В., Хованский А.Г. Теорема Римана-Роха для интегралов и сумм квазиполиномов по виртуальным многогранникам // Алгебра и анализ. 1992. Т. 4. № 4. C. $188-216$.

[86] Садов С. Ю. О динамической системе, возникшей из одной конечномерной аппроксимации уравнения Шрёдингера // Матем. заметки. 1994. Т. 56. № 3. С. 118-133.

[87] Садов С. Ю. Нормальная форма уравнения колебаний спутника в сингулярном случае // Матем. заметки. 1995. Т. 58. №5. С. 785-789. 
[88] Садов С. Ю. Метод усреднения для уравнения колебаний спутника при эксцентриситете, близком к единице // Материалы Международной конференции и Чебышёвских чтений, посвященных 175-летию со дня рождения П. Л. Чебышёва. Т. 2. М.: МГУ, 1996. С. 305-307.

[89] Sadov S. Yu. Singular normal form for a quasilinear ordinary differential equation // Nonlinear Anal. 1997. V. 30. № 8. P. 4973-4978.

[90] Самарский А. А., Галактионов В. А., Курдюмов С. П., Михайлов А. П. Режимы с обострением в задачах для квазилинейных параболических уравнений. М.: Наука, 1987.

[91] Шафаревич И.Р. Основы алгебраической геометрии. М.: Наука, 1972.

[92] Солеев А. Алгоритмы вычисления многогранников Ньютона // Докл. АН УзССР. 1982. № 5. C. $14-16$.

[93] Солеев А.Выделение ветвей аналитической кривой и многогранники Ньютона // Докл. AH CCCP. 1983. Т. 268. №6. С. 1305-1307.

[94] Солеев А., Арансон А. Вычисление многогранника и нормальных конусов его граней // Препринт № 36. М.: ИПМ РАН, 1994.

[95] Солеев А., Брюно А. Д. Многогранник Ньютона и системы Гамильтона // Вестн. МГУ. Сер. 1. Матем., мех. 1995. №6. С. 84-86.

[96] Ван-Дайк М. Методы возмущений в механике жидкости. М.: Мир, 1967.

[97] Варченко А.Н. Многогранник Ньютона и оценки осциллирующих интегралов // Функц. анализ и его прил. 1976. Т. 10. № 3. С. 13-38.

[98] Варченко А.Н., Хованский А.Г. Асимптотика интегралов по исчезающим циклам и многогранник Ньютона // Докл. АН СССР. 1985. Т. 283. № 3. С. 521-525.

[99] Васильев В.А. Асимптотика экспоненциальных интегралов, диаграмма Ньютона и классификация точек минимума // Функц. анализ и его прил. 1977. Т. 11. № 3. С. 1-11.

[100] Васильев М. М. О получении автомодельных решений уравнения движения вязкого теплопроводного газа // Препринт № 95. М.: ИПМ РАН, 1997.

[101] Васильев М. М. О расчете пограничного слоя на полубесконечной плоской пластине в стационарном потоке вязкого теплопроводного газа // Препринт № 43. М.: ИПМ РАН, 1999.

[102] Васильева А. Б., Бутузов В. Ф. Асимптотические методы в теории сингулярных возмущений. М.: Высшая школа, 1990.

[103] Веденяпин В.В., Мингалев О.В. Представления общих соотношений коммутации. Асимптотика спектра трех квантовых гамильонианов // Докл. АН. 1997. Т. 352. № 2. C. $155-158$

[104] Веденяпин В. В., Орлов Ю. Н. Асимптотика спектра квантовых гамильтонианов // Докл. АН. 1996. Т. 351. №4. С. 444-447.

[105] Веденяпин В.В., Орлов Ю. Н. О законах сохранения для полиномиальных гамильтонианов и для дискретных моделей уравнения Больцмана // ТМФ. 1999. Т. 121. № 2 . C. $307-315$.

[106] Виноградов А. М., Воробьев Е. М. Применение симметрий для нахождения точњых решений уравнения Заболоцкой-Хохлова // Акуст. журн. 1976. Т. 22. №1. С. 23-29.

[107] Волевич Л.Р.Энергетический метод в задаче Коши для дифференциальных операторов, корректных по И. Г. Петровскому // Труды ММО. 1974. Т. 31. С. 147-188.

[108] Волевич Л. Р., Гиндикин С. Г. Об одном классе гипоэллиптических полиномов // Матем. сб. 1968. Т. 75. №3. С. 400-416.

[109] Волевич Л. Р., Гиндикин С. Г. Многогранник Ньютона и локальная разрешимость линейных дифференциальных уравнений в частных производных // Труды ММО. 1985. T. 48. C. 211-262.

[110] Уокер Р. Алгебраические кривые. М.: ИЛ, 1952.

Институт прикладной математики им. М.В. Келдыша РАН

Поступила в редакцию

E-mail: bruno@spp.keldysh.ru

17.12.1999 